\title{
Comparison of Test and Finite Element Analysis for Two Full-Scale Helicopter Crash Tests
}

\author{
Martin S. Annett ${ }^{1}$ and Lucas G. Horta ${ }^{2}$ \\ NASA Langley Research Center, Hampton, VA, 23681
}

Finite element analyses have been performed for two full-scale crash tests of an MD-500 helicopter. The first crash test was conducted to evaluate the performance of a composite deployable energy absorber under combined flight loads. In the second crash test, the energy absorber was removed to establish the baseline loads. The use of an energy absorbing device reduced the impact acceleration levels by a factor of three. Accelerations and kinematic data collected from the crash tests were compared to analytical results. Details of the full-scale crash tests and development of the system-integrated finite element model are briefly described along with direct comparisons of acceleration magnitudes and durations for the first full-scale crash test. Because load levels were significantly different between tests, models developed for the purposes of predicting the overall system response with external energy absorbers were not adequate under more severe conditions seen in the second crash test. Relative error comparisons were inadequate to guide model calibration. A newly developed model calibration approach that includes uncertainty estimation, parameter sensitivity, impact shape orthogonality, and numerical optimization was used for the second full-scale crash test. The calibrated parameter set reduced 2-norm prediction error by $51 \%$ but did not improve impact shape orthogonality.

Nomenclature

$g_{i}(t) \quad$ scalar function with time variation

$i, k, I \quad$ integer indices

$m \quad$ number of measured locations

I number of impact shapes used in reconstruction

$p \quad$ parameter vector

$t \quad$ time

y analysis response vector

$y_{\theta} \quad$ test response vector

I identity matrix

$L \quad$ total number of time samples

$J \quad$ optimization metric

$M_{1} \quad$ calibration Metric 1

$M_{2} \quad$ calibration Metric 2

$N \quad$ number of solutions

$Q \quad$ scalar with 2-norm of response vector for analysis

$Q_{e} \quad$ scalar with 2-norm of response vector for test

$W \quad$ weighting matrix

$\delta_{i} \quad i^{\text {th }}$ impact shape contribution to response

$\Delta T \quad$ sample time

${ }_{1}^{1}$ Aerospace Engineer, Structural Dynamics Branch, Mail Stop 495, AIA A member

2 Aerospace Engineer, Structural Dynamics Branch, Mail Stop 230, AIA A Associate Fellow 1

American Institute of Aeronautics and Astronautics 


\begin{tabular}{|c|c|}
\hline \multirow[t]{2}{*}{$\sigma_{i}$} & $t^{\text {th }}$ singular value \\
\hline & $I^{\text {th }}$ impact shape vector at measured locations \\
\hline$\breve{\Psi}, \breve{\Phi}$ & normalized impact shape matrices \\
\hline Prob & probability \\
\hline $\max$ & maximum value \\
\hline & relational operators (less than, or, greater than) \\
\hline & maximum of all maxima \\
\hline ( & minimum of all minima \\
\hline()$^{T}$ & matrix transpose \\
\hline
\end{tabular}

\section{Introduction}

$\mathrm{C}$ rashworthiness is defined as the ability of a vehicle structure to withstand impact loads, sustain occupiable volume, and limit loads on the occupants. Throughout the rotorcraft community, novel crashworthy features are being researched and incorporated that significantly absorb kinetic energy for mild to severe-but-survivable impacts. These concepts fundamentally require large inelastic deformations to be effective. External energy absorbing systems, such as airbags and crushable composite cushions, must be easily stowed and rapidly deployed without affecting operability. Internal systems, including load limiting seat struts and crushable subfloors, are currently in operation but are limited in effectiveness by available space.

The NASA Subsonic Rotary Wing Project (SRW) is sponsoring research to evaluate new materials and structural concepts to improve rotorcraft crashworthiness and to increase occupant survivability (Ref.1). As part of this effort, two full-scale helicopter tests were conducted to study structural concepts for crashworthiness, to develop and validate component models to predict energy absorption performance, and lastly to validate system-integrated finite element models. In the first full-scale test, an advanced composite energy absorber concept, referred to as the Deployable Energy Absorber (DEA), was evaluated to assess performance. The concept, conceived and patented by Dr. Sotiris Kellas of NASA Langley Research Center (LaRC) (Ref.2], is a Kevlar/epoxy honeycomb structure. To assess the full-scale landing loads, a second test was conducted that did not include the DEA. Results for both of these tests are presented in this report.

Attempts to compare aerospace vehicle-level simulations with full-scale testing originated in the late 1970's with the KRASH analysis program (Ref. 3). The airframe structure was represented as a framework of concentrated mass and bar elements and relied heavily on semi-empirical data to tailor the model response. A US Army Advanced Composite Airframe Program (ACAP) helicopter crash test was compared initially to KRASH simulations by Cronkhite and Mazza (Ref.4). Throughout the 1980's, codes that apply explicit time integration techniques to solve transient dynamic problems and provide the capability to handle both material and geometric nonlinearities became commonplace. Simulations with the explicit finite element analysis program MSC/DYTRAN were compared to the ACAP test by Jackson et al. (Ref.5). KRASH was coupled with MSC/DYTRAN to provide a hybrid computational framework for correlation with UH-1 helicopter water impact tests (Ref.6). Recently, LS-DYNA has become the predominant commercial code for conducting crashworthiness simulations due to its robust contact algorithms and extensive material library (Ref. 7). Jackson and Fasanella compared results from a vertical drop test of an ATR42-300 fixed wing commuter aircraft with simulation using LS-DYNA (Ref.8).

Data collected from the full-scale MD-500 helicopter crash tests are crucial for validation of a system-integrated helicopter finite element model (FEM) (Ref. 9] and assessment of the model's overall predictive capability. System-integrated impact simulations have the advantage of accounting for highly nonlinear deformation, contact loading, and material response exhibited during impact. Interactions between critical components of the airframe, including skid gear, subfloor, seats, and DEA, can be simulated. Modeling detailed representations of the frame, seats, restraints, and occupants into a single FEM is commonplace within the automotive crashworthiness community. Prior to the mid 1990's, most impact analyses were sequentially coupled, with vehicle model acceleration responses defined as pulse inputs for occupant, seat, and restraint models. Interaction between the 
vehicle and occupant models required simplifying assumptions, potentially reducing the accuracy of computed injury criteria. Recent advancements in computing capacity have permitted a high level of model fidelity to be feasible.

The system-integrated FEM model for the helicopter test reported here was originally developed prior to full-scale testing to provide pre-test predictions and to aid in test planning. A robust FEM is practical for assessing vehicle responses and occupant loads under multi-terrain impacts and varying impact velocities and attitudes. Ultimately, the goal is to develop and assess modeling tools for crash safety certification by analysis. For the military, the standard for light fixed wing and rotary wing crash resistance (MIL-STD-1290A (AV)) details seven crash impact design scenarios and specifies occupant seat acceleration limits and occupied volume reduction constraints (Ref.10). These design conditions are intended to encompass all weight classes and account for only two impact surfaces, rigid and plowed soil.

The analytical capabilities available when this standard was established were limited, therefore, it was expected that crash testing would be primarily used to determine compliance. Ever since, more crashworthy features have been implemented into designs, more mishap data has been accumulated, and modeling and prediction tools for crashworthiness and injury biomechanics have improved. Efforts are underway to revisit the existing requirements and generate a full spectrum of crashworthiness criteria that accounts for weight class, operating conditions, and impact conditions (Ref. 11). Survivability envelopes for a range of velocities, attitudes, and terrains can be developed from both tests and system integrated models with a high degree of confidence. As the technology evolves to efficiently incorporate more modeling and simulation into the design process, next generation rotorcraft will contain more crashworthy features without sacrificing weight and performance.

Verification and validation of computational models involves quantitatively assessing the accuracy of the analytical predictions. Verification of models involves determining if the mathematical realization of the equation of motion is accurately implemented, whereas the validation phase involves ensuring that the physics of the problem agrees with the problem at hand (Ref. 12). Once these first two phases are completed, model calibration is undertaken to adjust the model parameters to reconcile analysis with test. Because this work relies on commercial finite element codes that are customarily used for this class of problems, it is assumed that verification of the code is accounted for by the code vendor. Hence this work is strictly focused on validation and model calibration.

In the following sections, the experimental program and finite element model development and validation efforts will be discussed. Calibration of the finite element model is performed. For calibration, both heuristic and quantitative methods are used to identify modeling deficiencies, evaluate parameter importance, and propose required model changes. Calibration requires multiple model simulations with a variety of parameter inputs. Therefore, calibration approaches are adopted that are computationally affordable yet include sufficient modeling detail.

\section{Full-Scale Crash Test Description}

Full-scale crash tests of the MD-500 helicopter were performed at NASA Langley's Landing and Impact Research Facility in November 2009 and March 2010. Figure 1 shows the facility and a notional schematic of a swing test. Target impact conditions were 26 -ft/sec vertical and $40-\mathrm{ft} / \mathrm{sec}$ horizontal while maintaining zero pitch, roll, and yaw attitude. Although this impact condition is severe, it is still considered survivable. The test was conducted by suspending the helicopter from the gantry structure using two sets of cables: pullback cables and swing cables. These cables were attached to the airframe at hard points that enable the helicopter to be lifted through its center of gravity (CG). During the test, the airframe was lifted using the pullback cables to a specified height and pyrotechnically released following a countdown. Swing cables were configured to form a parallelogram to minimize pitch angular velocity during the pendulum swing prior to impact. Just prior to ground contact, the supporting cables were pyrotechnically separated.

A photograph of the MD-500E helicopter, manufactured by MD Helicopters, Inc. of Mesa Arizona, is shown in Figure 2. Currently the MD-500E is used as a general-purpose utility and executive transport helicopter for both military and civilian applications. The MD-500 helicopter is designed to seat four occupants, two crew and two passengers. The test article is shown in Figure 2h. Occupants were placed in standard seats and restrained using 
four-point harnesses for the crew and three point harnesses for the passengers. Seats consisted of a framework of aluminum tubing and nylon mesh fabric stretched over the frames to form a seat pan and seat back.

The target mass for the test article was set to $2,900 \mathrm{lb}$, which is roughly the maximum gross takeoff weight for the MD-500E. Ballast mass was distributed onto the test article by adding steel tubing for swing cabling fixtures, steel plates and tubing to represent rotor and tail mass, and data acquisition support hardware to represent the transmission. Sand bags were placed in the subfloor region to account for fuel mass. The total weights of the test article with and without the DEA were $2,940 \mathrm{lb}$ and 2,906 lb, respectively.

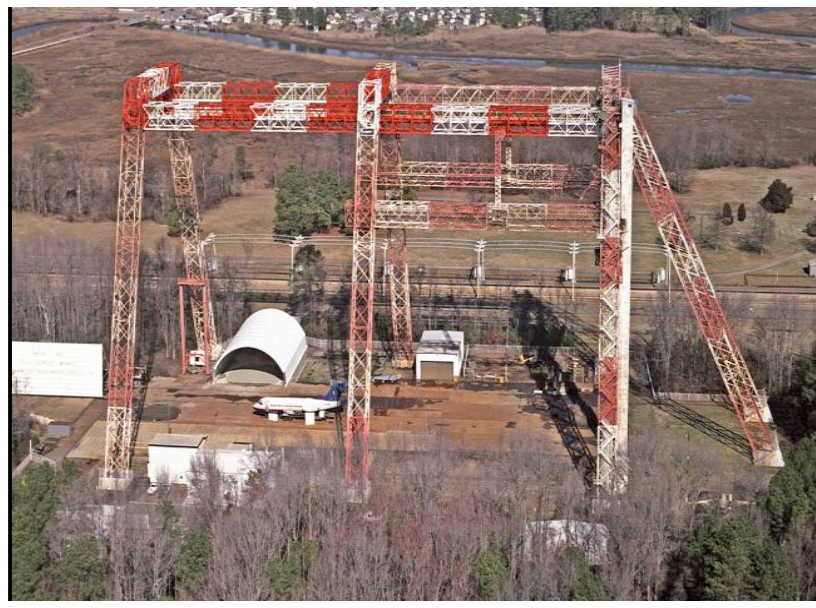

(a) Photograph of LandIR

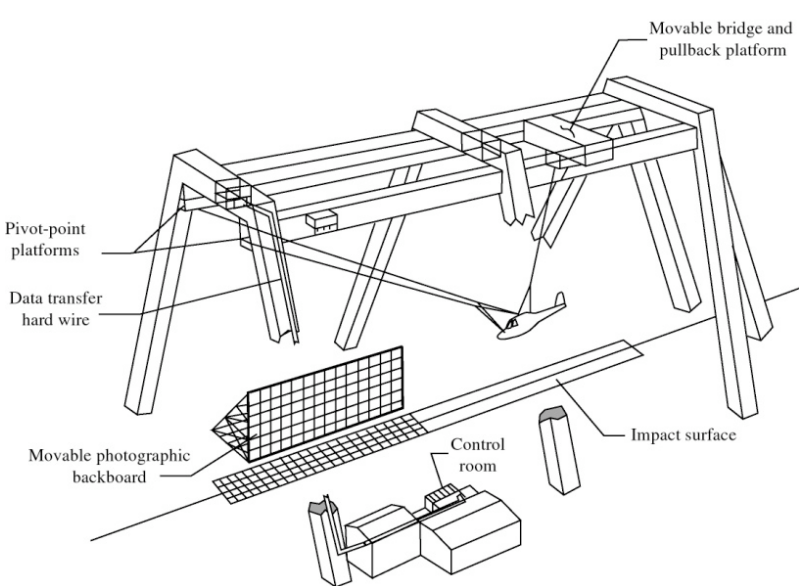

(b) Schematic of LandIR

Figure 1. NASA Langley Research Center-Landing and Impact Research Facility

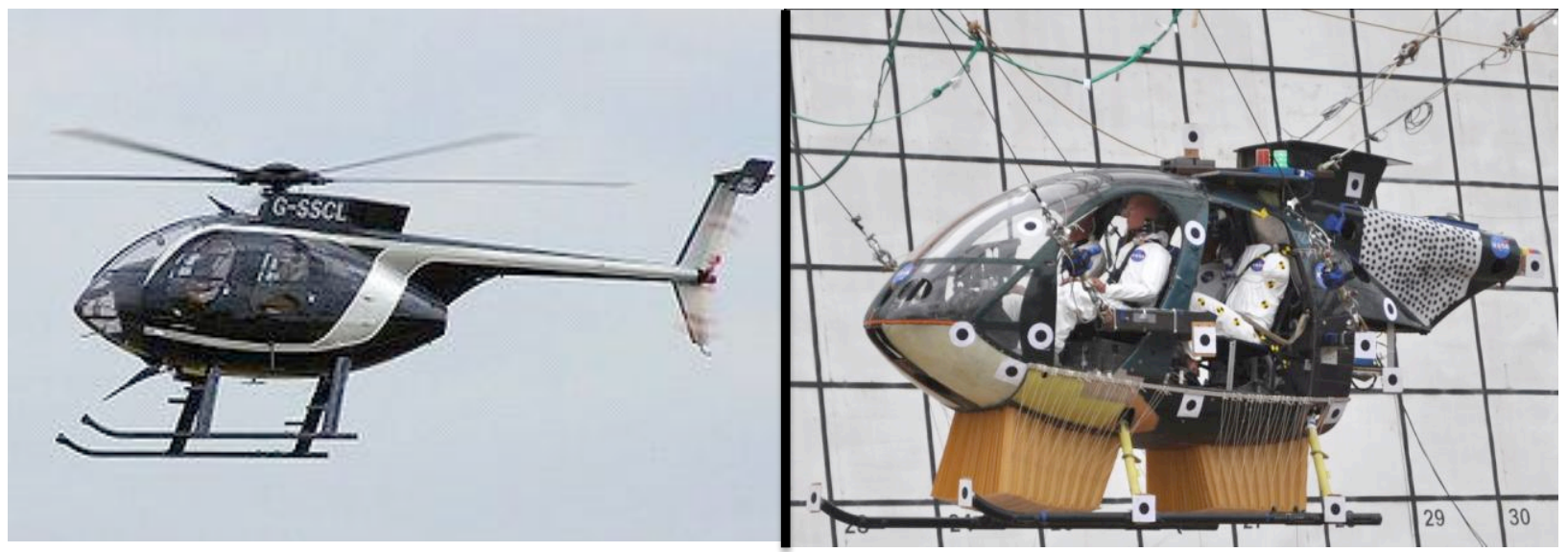

(a) MD-500E helicopter

(b) MD-500 Crash Test Article

Figure 2. MD-500 Helicopter

Four instrumented Anthropomorphic Test Devices (ATD) were used to represent two crew and two passengers. The pilot in the front left crew position was a 50th percentile Hybrid III male ATD with a straight lumbar spine similar to the Hybrid II. The co-pilot in the front right crew position was a 50th percentile Hybrid II male ATD, and the rear passenger on the left side was a 50th percentile Hybrid II male ATD. The Hybrid II and III ATDs weigh $180 \mathrm{lb}$. For the right rear passenger a specialized Human Surrogate Torso Model (HSTM) developed by The Johns Hopkins University Applied Physics Laboratory (APL) was used. This biofidelic HSTM contains detailed 
representations of thoracic organs, skeletal structure, and soft tissue and is mated to the pelvis and legs of a 50th percentile Hybrid III male ATD (Ref.13. The weight of the HSTM/Hybrid III ATD is $170 \mathrm{lb}$.

The fuselage and skid gear were instrumented with a combination of strain gages and accelerometers. ATD instrumentation included head, chest, and pelvic accelerometers, lumbar load cells, restraint load cells, and pressure gages. A total of 160 channels of data were collected at a sample rate of $10,000 \mathrm{~Hz}$. In addition, measurements of vehicle kinematics were taken using two and three dimensional photogrammetry. For the purposes of this study and test analysis comparison, 24 sensor locations containing either triaxial and uniaxial accelerometers, totaling 37 channels, were used.

A detailed description of the test results is provided in Ref. 14. The impact conditions for the two full-scale helicopter tests are summarized in Table 1. Note that the pitch and yaw attitudes for the first test were off-nominal. It was determined that the variations in the distribution of swing cable tension loads prior to release introduced rotational motion upon release. Several pre-lift tests were conducted prior to the second full-scale crash test to determine proper alignment procedures for cable loads which resulted in impact conditions closer to nominal.

Table 1. Full-Scale Test Impact Conditions

\begin{tabular}{|c|c|c|c|c|}
\hline \multicolumn{2}{|c|}{ Test Parameters } & $\begin{array}{c}\text { Nominal } \\
\text { Conditions }\end{array}$ & $\begin{array}{c}\text { MD-500 with } \\
\text { DEA }\end{array}$ & $\begin{array}{c}\text { MD-500 } \\
\text { Without DEA }\end{array}$ \\
\hline \multicolumn{2}{|c|}{ Vehicle Weight (Ib) } & 2,900 & 2,940 & 2,906 \\
\hline \multirow{3}{*}{$\begin{array}{l}\text { Linear } \\
\text { Velocity } \\
\text { (ft/sec) }\end{array}$} & Forward & 40. & 38.8 & 39.1 \\
\hline & Vertical & 26. & 25.6 & 24.1 \\
\hline & Lateral & 0 & 0.5 & 0.6 \\
\hline \multirow{3}{*}{$\begin{array}{l}\text { Attitude } \\
\text { (deg) }\end{array}$} & Pitch & 0 & -5.69 & -6.2 \\
\hline & Roll & 0 & 7.04 & 1.9 \\
\hline & Yaw & 0 & 9.3 & 2.1 \\
\hline \multirow{3}{*}{$\begin{array}{l}\text { Angular } \\
\text { Velocity } \\
\text { (deg/sec) }\end{array}$} & Pitch Rate & 0 & 0.44 & 0.54 \\
\hline & Roll Rate & 0 & 1.11 & 0.68 \\
\hline & Yaw Rate & 0 & 4.82 & 1.65 \\
\hline
\end{tabular}

\section{Full Scale Crash Test Results}

Figure 3 shows test sequence photos for the crash test with the DEA. Picture 1 shows the helicopter approximately $30 \mathrm{~ms}$ before impact, pitched down and with some slight yaw. Picture 2 shows the helicopter at the point of first skid gear impact when the right gear touches the ground. Picture 3 shows the point of maximum DEA crushing, and picture 4 shows post-impact rebound. The front right-side skid gear impacted the ground first, which was caused by the yaw and roll introduced during the swing. At the point of maximum crushing of the DEA (picture 3 ), the helicopter straightened out to show almost no pitch. After the point of maximum crush, the nose pitched forward on rebound, and the Hybrid ATD heads and torsos flailed forward and to the left.

Overall, the damage to the test article was minor. Impact occurred initially on the front right skid gear. Slight tears in the skin above the fuselage opening were evident for both skid gears. The DEA restraint support rail impeded the gear from additional movement with the result that the right gear bent along the rail. Damage along the fuselage belly was limited to the right front section of the belly forward of the front bulkhead. The subfloor and airframe were considered intact, and minimal repair work was required on the forward keel beam and belly to prepare the test article for the destructive crash test without DEA.

Figure 4 shows a sequence of photos for the test without the DEA. Picture 1 shows the helicopter before impact. Picture 2 shows the helicopter at the point of first skid gear impact. As with the MD-500 crash test without DEA, the right gear impacted the ground first, but the amount of yaw and roll was lower than the test with the DEA. The fuselage belly impacted the ground approximately $80 \mathrm{~ms}$ after gear impact, and the highest vertical deceleration loads were seen thereafter. Picture 3 shows the point of maximum vertical displacement, where the helicopter maintained a slight nose down pitch. Picture 4 shows minor post-impact rebound. After the point of maximum subfloor deformation, the nose pitched forward on rebound, and flailing of the ATD heads and torsos occurred. 


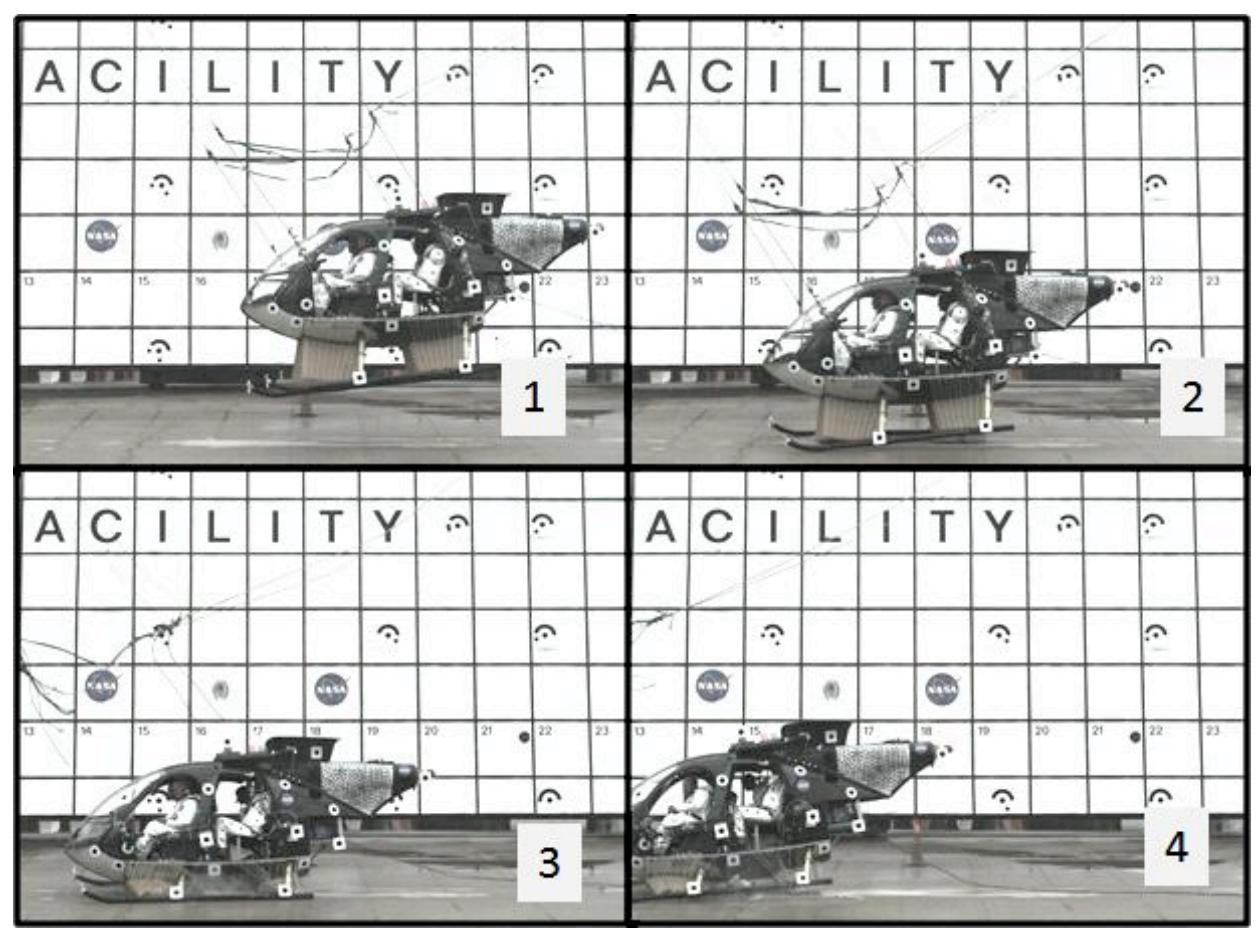

Figure 3. Test sequence from south camera, crash test with DEA.

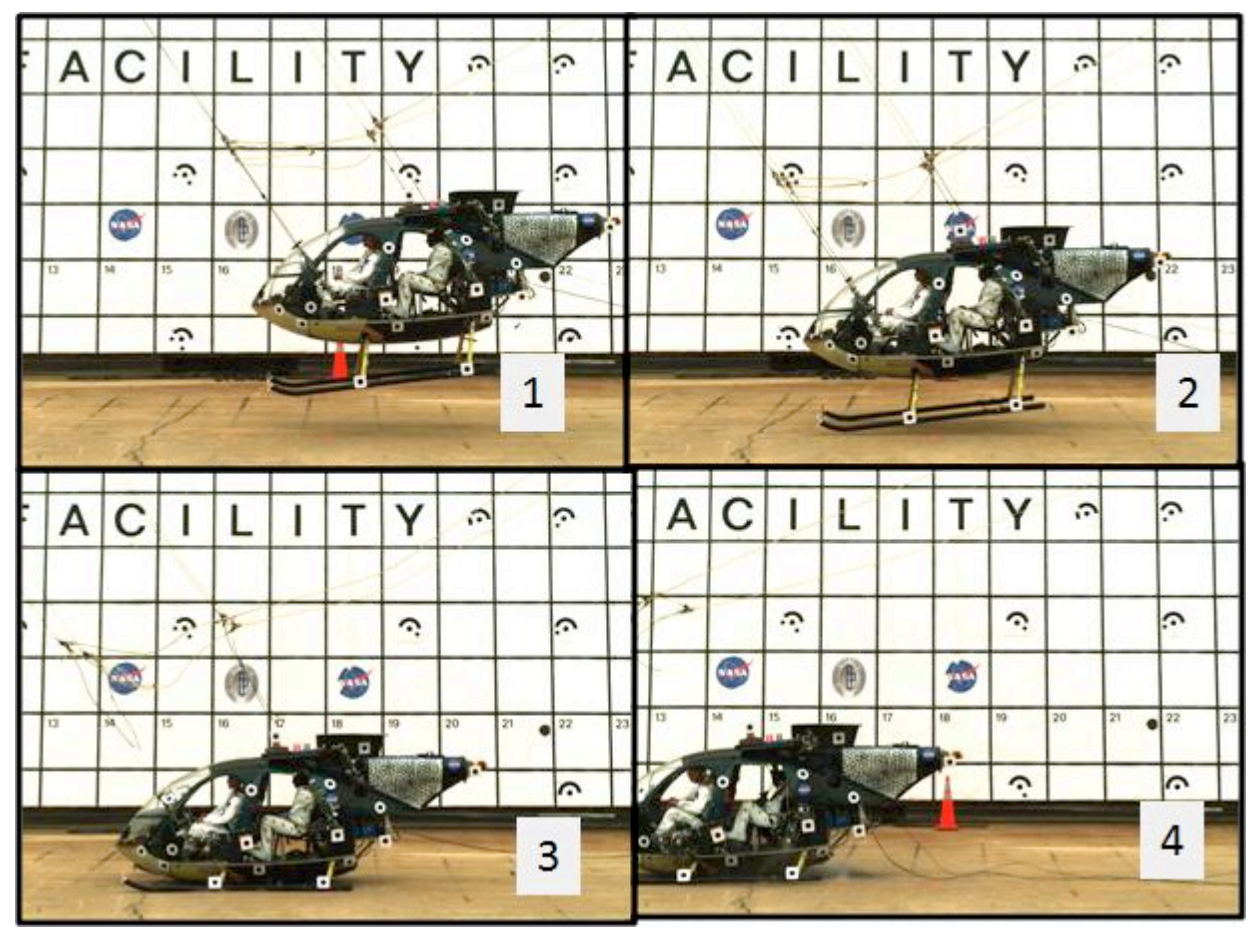

Figure 4. Test sequence from south camera, crash test without DEA. 


\section{LS-DYNA Finite Element Model}

A full description of the system-integrated LS-DYNA FEM for the MD-500, shown in Figure 5 , is included in Ref. 9. A computer-aided design model of the MD-500 fuselage was provided by the U. S. Army Aviation Technology Directorate and consisted of surface representations of the fuselage, bulkheads, seat supports, and floor. Additionally, the skid gear, subfloor, and secondary frames and stiffeners were modeled from hand measurements. The crew and passenger seats were modeled with target tracking 3-D photogrammetric techniques in which photogrammetric point clouds were converted to parametric surfaces and finite element meshes.

The FEM is primarily composed of shell elements, representing airframe skins, frames, stiffeners, skid gear, and DEAs. Material properties for the fuselage are based on the MD-500 Structural Repair Manual (Ref. 15. The fuselage is primarily Aluminum 2024-T3 with elastoplastic properties. The nose is composed of fiberglass, and the engine fairing is composed of Kevlar fabric. Crush tube struts are used to attached the skid gear to the fuselage and to distribute the landing and impact loads. These tube struts are modeled as one-dimensional spring elements that transmit axial loads and bending moments. Ballast and non-structural components are represented in the FEM with concentrated mass elements.

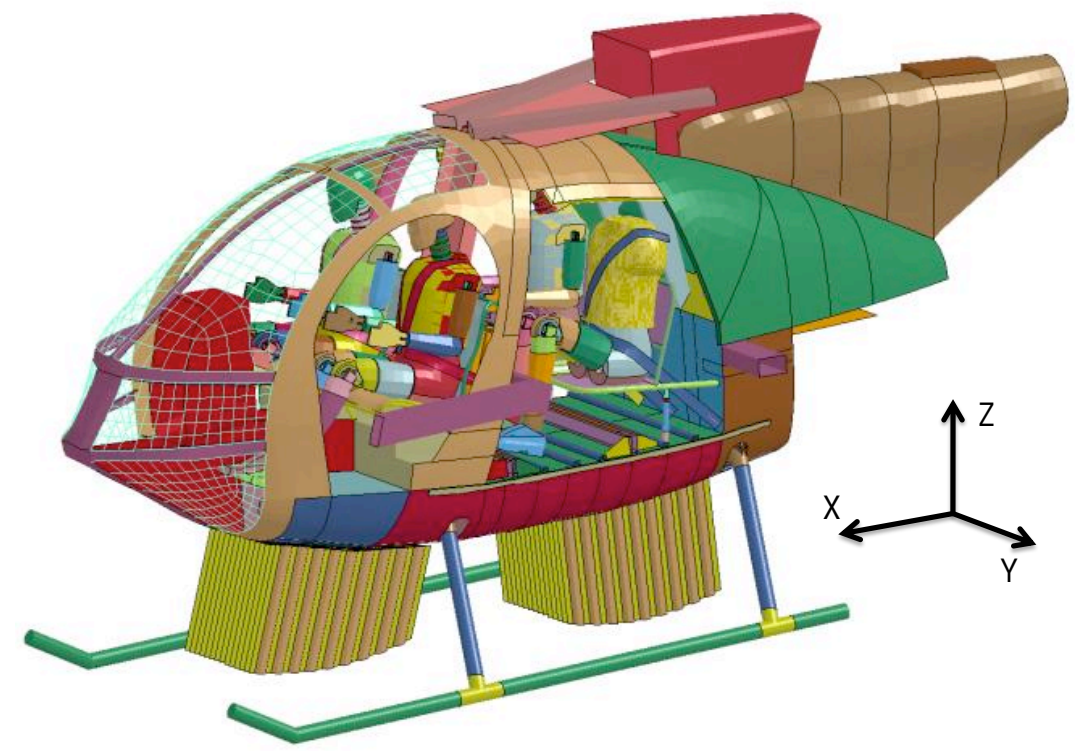

Figure 5. MD-500 FEM with DEA

\section{A. DEA Design and Modeling}

A key component evaluated in the first impact test was the DEA. This design allows for the DEA to be stowed flat external to the fuselage belly and deployed to form the hexagonal cell walls as notionally shown in Figure 6. In this configuration, the DEA is loaded along the stiff cell axis causing the cell to yield plastically under load and thereby absorb energy. The cell walls fold to form a controlled accordion-like pattern (see Figure 6h). The effectiveness of the DEA was evaluated using a hierarchical approach beginning with material coupon static tests, progressing to sub-component static and dynamic tests, and culminating with full-scale crash testing. 


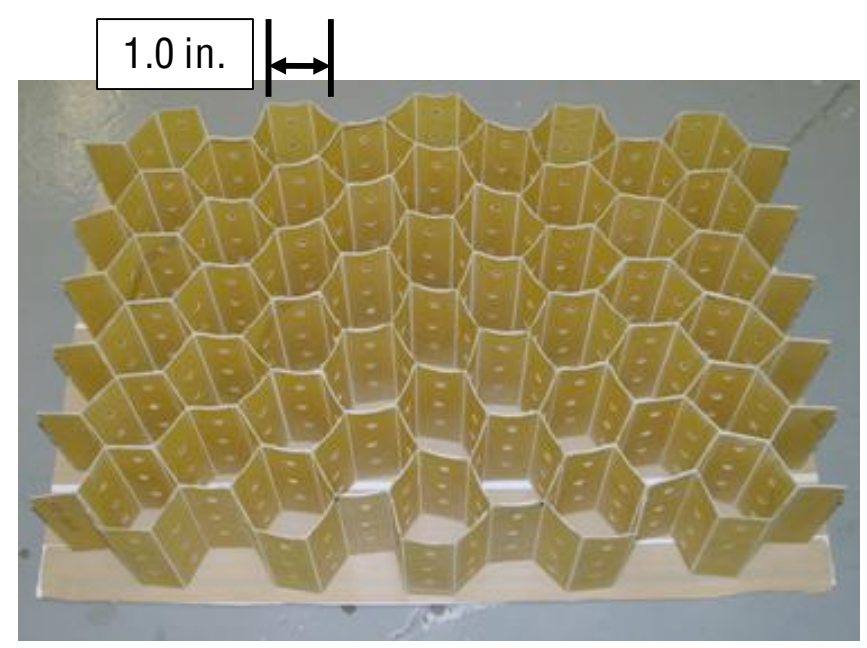

(a) DEA in deployed configuration

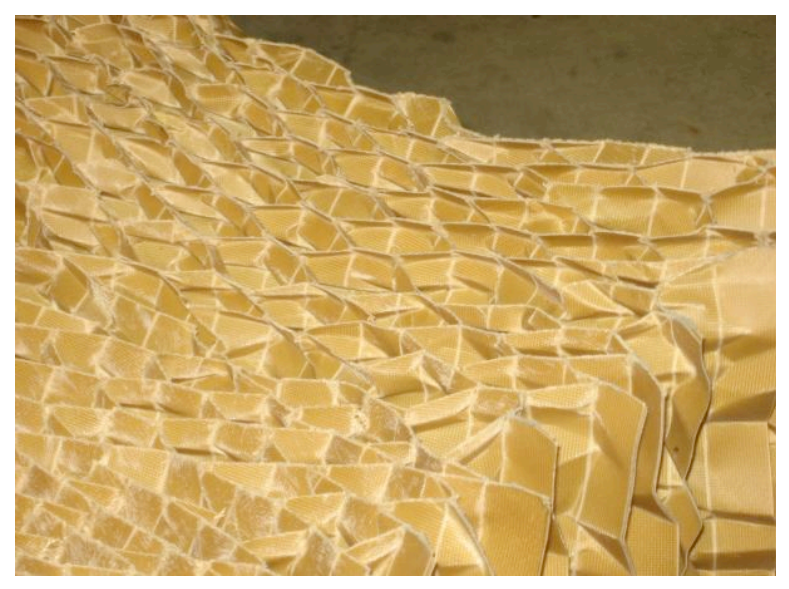

(b) Post-impact DEA deformation

Figure 6. DEA Concept

A schematic of the shell-based DEA model is illustrated in Figure 7. Convergence studies reported in Ref. 16 revealed that the maximum acceptable DEA element length was approximately 0.3 inches to replicate the folding patterns accurately. The material model is elastic and piecewise linear plastic with a Young's Modulus of $340,000 \mathrm{psi}$ and initial yield stress of 7,500 psi. The yield stress versus plastic strain curve is plotted in Figure $7 \mathrm{p}$.

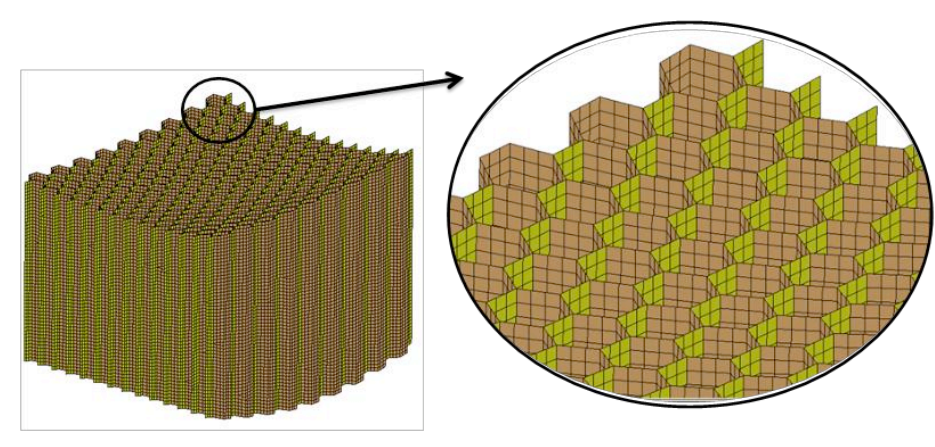

(a) Shell Based DEA FEA

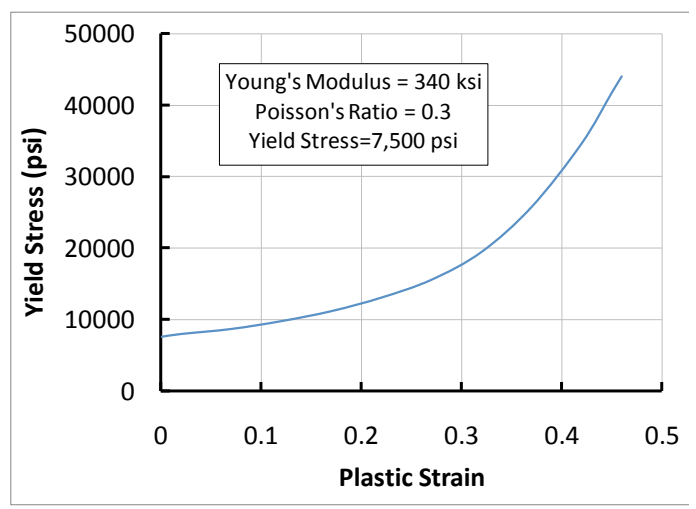

(b) DEA Material Properties

Figure 7. Shell-Based DEA Model and Properties

B. Anthropomorphic Test Device Modeling

A model of the 50th percentile Hybrid III male, denoted as the LSTC Hybrid III FEM (Ref.17), was used for the Hybrid II and III ATDs (Figure 8). These models contain mostly rigid representations of the ATD components. However, the ribcage, neck, jacket, and pelvis are deformable. Springs and dampers were used in the neck and limb joints to model flexibility. To position the ATD, the FEMs were imported using the LS-DYNA pre-processor. Each ATD FEM contains 4,295 elements.

The Hybrid II and III ATDs used in the crash tests are notably different than what is characterized with the LSTC Hybrid III FEM. The LSTC Hybrid III FEM has been calibrated for automotive frontal impact conditions, 
with emphasis on capturing head/neck and chest kinematics. For testing in aerospace applications, the Hybrid II and III ATDs contain straight lumbar spines, whereas the LSTC Hybrid III FEM includes the automotive curved spine. Moreover, the LSTC Hybrid III FEM does not contain an abdominal insert, which becomes a potential load path from the pelvis to the ribcage under high vertical loading. Therefore, it is understood that the internal responses of the pelvic and lumbar region with the LSTC Hybrid III FEM are not accurate. Results reported in Refs. 18. and 19 support this finding. The mass distribution of the LSTC Hybrid III FEM is accurate provided there is limited torso flail due to the presence of shoulder restraints. Capturing the ATD/seat impact, and its subsequent rebound and effective mass decoupling, is critical for determining the loads in the airframe. The LSTC Hybrid III FEM captures this behavior and is therefore an upgrade over simply using lumped mass representations.

A reduced human torso FEM was constructed and adapted from APL's detailed Human Torso Finite Element Model (HTFEM) (Ref. 13). The organs and soft tissue are represented by solid silicone elements. The sternum, ribcage, vertebrae and scapula are modeled with fiberglass bar and shell elements. The bar and shell elements are embedded within the solid elements and coupled with constraint algorithms. The reduced HTFEM was attached to the LSTC Hybrid III FEM pelvis and legs and the total model size is 8,034 elements. The reduced HTFEM is depicted in Figure 8p. The pilot and co-pilot FEMs are restrained with four-point harnesses, and the passenger FEM and reduced HTFEM are restrained with three-point harnesses. Seatbelt shell elements were contoured to the torso and pelvis.

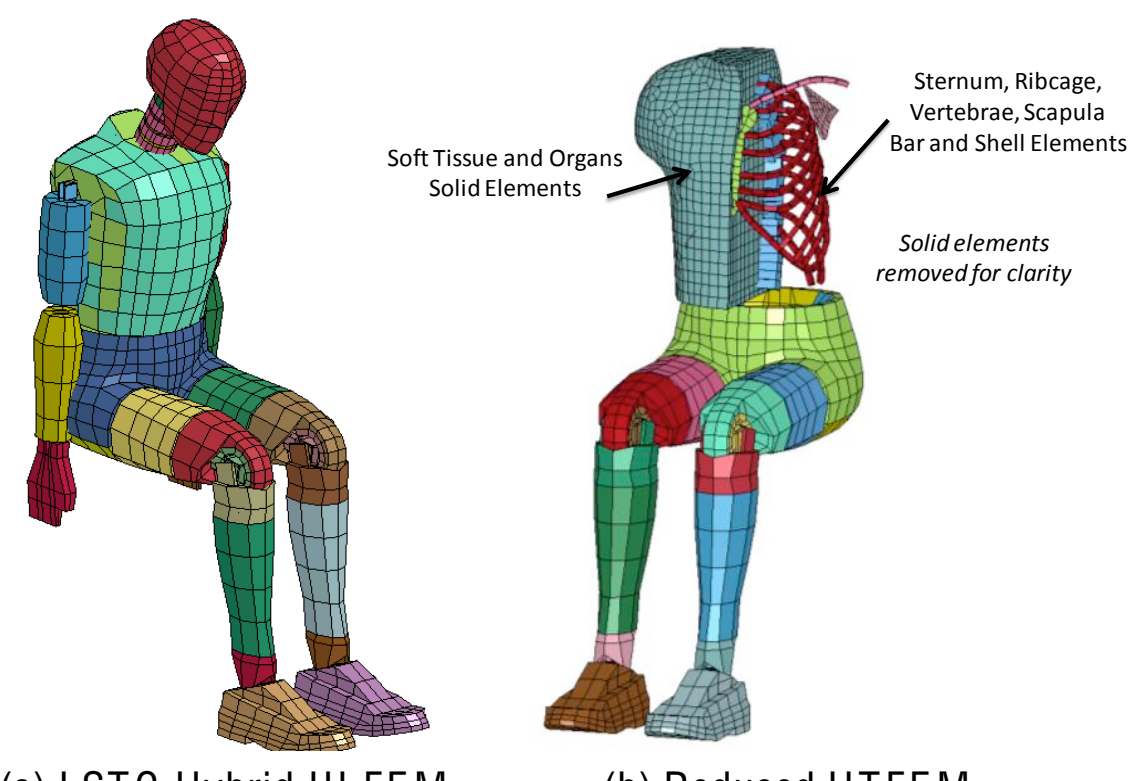

(a) LSTC Hybrid III FEM

(b) Reduced HTFEM

Figure 8. ATD Finite Element Models

The MD-500 FEM with the DEA has approximately 400,000 elements in total, with 266,000 elements representing the DEA. This model size is commensurate with automotive crash model sizes. There are continually tradeoffs when refining models that use explicit finite element techniques where stability is conditionally enforced if the time steps are sufficiently small. The time step is a function of the shortest element dimension; therefore, for more refined meshes, the time steps decrease and overall runtime increases. For simulation times of 0.2 seconds, the system-integrated MD-500 model runtime is approximately 24 hours.

\section{Test/Analysis Comparison of Crash Test with DEA}

A detailed summary of the test responses is provided in Ref.14. The test impact orientation and deformation at peak load is shown for test and analysis in Figure 9. Qualitatively, the global deformation pattern of the deployable energy absorber is similar to the deformation observed from the high speed video, primarily folding on the right side and crushing on the left side. Consequently, the locations where the DEA cells simply buckled transferred higher impact loads than those locations where crushing occurred. However, because damage to the front right side was not 
evident in the analysis, these regions of folding and crushing do not correspond between test and analysis. Within the simulation, dimpling of the skin occurred in the region above the rear DEA, whereas the post-test inspection revealed no damage. The indiscriminate behavior of DEA folding, crushing and sliding along the belly was due to the presence of lateral and horizontal loading and was only partly captured with the shell-based DEA model.

Despite the qualitative differences between local deformation patterns, the overall response of the airframe is in reasonable agreement. Nodal accelerations at two critical locations, the pilot seat box and the centerline of the floor beneath the passenger seats, are compared to accelerometer output. The reference coordinate system for the simulation and the test are fixed along the floor surface. The axis perpendicular to the floor represents vertical. Predictions of ATD responses such as pelvic accelerations and lumbar loads are disregarded because the load paths and compliance of the pelvis and lumbar regions are inaccurate. Instead, for purposes of evaluation of occupant injury criteria such as Eiband, Dynamic Response Index, and Brinkley (Refs.20\#22), the responses for the pilot seat box and passenger floor should be used as inputs.

The pilot seat box vertical accelerations are plotted in Figure 10, and the passenger floor accelerations in Figure 11. The data comparisons are plotted for 0.2 seconds. All acceleration data are low-pass filtered with a second order Butterworth $60 \mathrm{~Hz}$ filter. As expected, the vertical acceleration responses of the airframe are effectively trapezoidal with durations of roughly $0.12 \mathrm{~s}$. Note that the DEA performs as a load-limiting shock absorber, regulating the loads between 10 and $15 \mathrm{~g}$ and lengthening the duration of the imparted loads through crushing and folding. Because the airframe rebounded before full compaction of the DEA could occur, no abrupt increases in the imparted loads were observed.

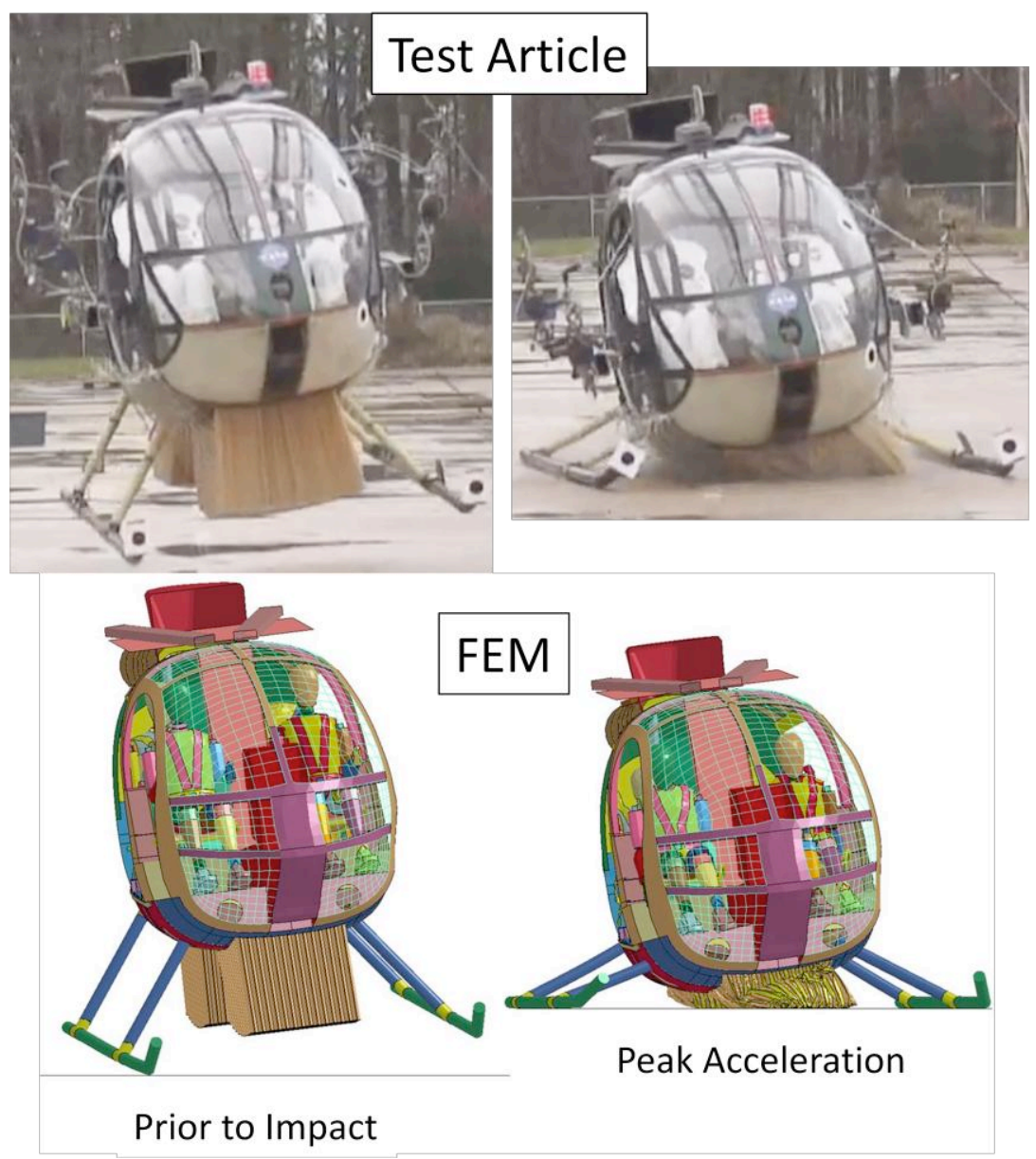

Figure 9. MD-500 FEM deformation 


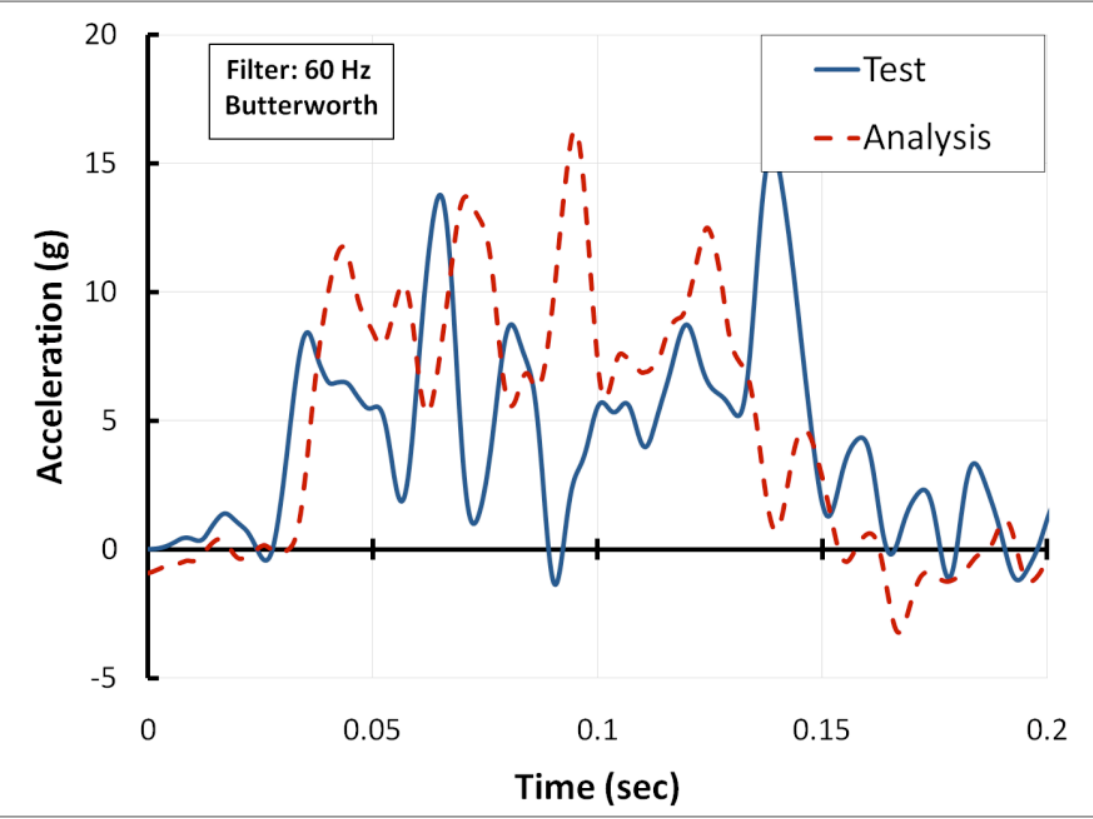

Figure 10. Comparison of test and analysis, pilot seat box vertical acceleration, crash test with DEA

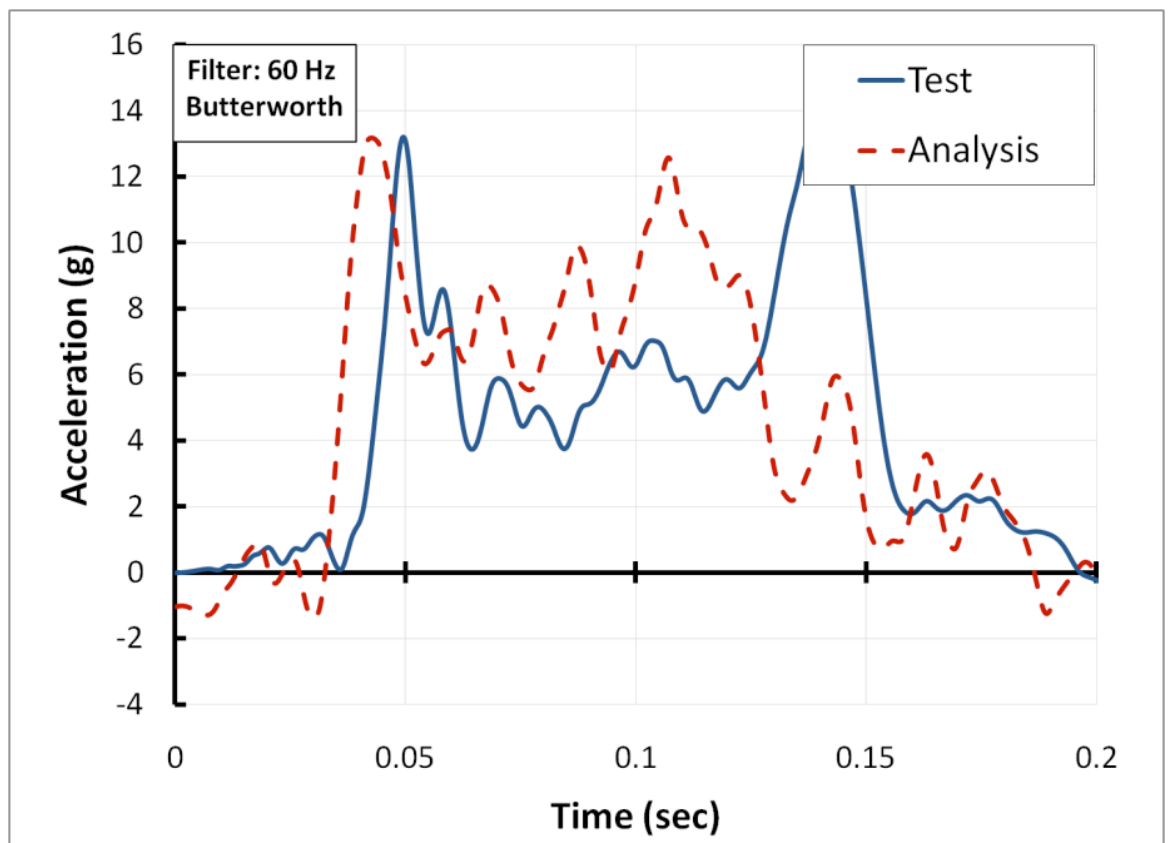

Figure 11. Comparison of test and analysis, passenger floor vertical acceleration, crash test with DEA

\section{Model Calibration Approach for Full-Scale Test Without DEA}

Typically, validation comparisons between test and analysis are both qualitative and quantitative. One qualitative approach, only possible by the use of high-fidelity finite element models, is to compare post-impact airframe deformations and regions of damage. Quantitatively, output time history responses such as acceleration, velocity, strain, and pressure can be compared between sensor locations and their respective model nodes or elements. 
Relative errors for magnitude, time of arrival, and pulse duration can be computed. For a more computationally rigorous formulation, the approach initially discussed in Ref.23, is applied to the second test without the DEA.

Fundamental to the success of the model calibration effort is whether the model can predict the observed behavior in the presence of modeling uncertainty. Although there is no universally accepted metric to judge model adequacy, the approach followed with the second test uses uncertainty propagation and quantification to assess model adequacy. The first step in this approach is parameter selection (parameters being in this case material properties, structural dimensions, initial conditions etc.,) which relies heavily on the analyst's knowledge and familiarity with the model and assumptions. After an initial parameter set is selected, uncertainty models to prescribe parameter variations must be defined with the aid of empirical data or oftentimes one must resort to engineering judgment. With an initial parameter set and an uncertainty model at hand, parameter importance is assessed using uncertainty propagation. Parameter values in this paper are created using the Halton (Ref. 24] deterministic sampling technique. Time history results are processed to compute the metrics and to assess variability. A by-product of this step produces variance-based sensitivity results which are used to rank the parameters. In the end, adequacy of the parameter set is judged based on the probability of one being able to reconcile test with analysis.

Analysis of variance (ANOVA) is used for parameter sensitivity. In classical ANOVA studies, data is collected from multiple experiments while varying all parameters (factors) and also while varying one parameter at a time. These results are then used to quantify the output response variance due to variations of a particular parameter, as compared to the total output variance when varying all the parameters simultaneously. The ratio of these two variance contributions is a direct measure of the parameter importance. Sobol et al. (Ref. 25) and others (Refs. 26/28) have studied the problem as a means to obtain global sensitivity estimates using variance-based methods. To compute sensitivity using these variance based methods, one must be able to compute many response predictions as parameters are varied. For this paper, after a suitable set of LS-DYNA solutions are obtained, response surface surrogates are used to estimate additional solutions.

The Extended Radial Basis Functions (ERBF) response surface method, as described by Mullur (Refs. 29,30), is used to estimate time histories. In this adaptive response surface approach, the total number of response surface parameters computed equals $N\left(3 n_{p}+1\right)$, where $n_{\mathrm{p}}$ is the number of parameters and $N$ is the number of LS-DYNA solutions. The user must also prescribe two additional parameters: 1 ) the order of a local polynomial (set to 2 in the present case), and 2) a smoothness parameter (set to 0.15 here). Finally, the radial basis function is chosen to be an exponentially decaying function $e^{-\left(p-p_{i}\right)^{2} / 2 r_{c}^{2}}$ with characteristic radius $r_{c}$ set to 0.15 . A distinction with this response surface implementation is that ERBF is used to predict full time histories, as opposed to just extreme values. In addition, ERBF is able to match the responses with prediction errors of order $10^{-10}$ at the points used to create the surrogate.

Uncertainty propagation is conducted to evaluate uncertainty bounds and to gage the ability of the model to explain the observed behavior. The statistics of the 2-norm of a response vector between test and analysis are compared. An important benefit of using this metric is that it provides for a direct measure of multi-dimensional closeness of two models. In addition, when tracked as a function of time, closeness is quantified at each time step. Because parameters are uncertain, statistical measures of the metric need to be used to conduct assessments. With limited information about parameter uncertainty, a uniform distribution function, which is the least informative distribution function, is the most appropriate representation to model parameter uncertainty. This uncertainty model is used to create a family of $N$ equally probable parameter vectors, where $N$ is a scalar arbitrarily selected. From the perspective of a user, it is important to know the probability of being able to reconcile measured data with predictions, given a particular model for the structure and parameter uncertainty. To this end, let

be a scalar time varying function of the 2-norm of the system response vector $v$, using parameter vector $p$ at time $t$. Furthermore, let $\underline{\sigma}(t)=\min _{\forall p} Q(t, p)$ be the minimum value over all parameter variations, and let $\bar{\sigma}(t)=\max _{\forall p} Q(t, p)$ be the maximum value. Using these definitions and $N$ LS-DYNA solutions, a calibration metric used to bound the probability of test values falling outside the analysis bounds is;

$$
M_{1}=\operatorname{Prob}\left(\left[\underline{\sigma}(t)<Q_{e}(t)\right] \vee\left[Q_{e}(t)>\bar{\sigma}(t)\right]\right)<<1 / N
$$


where $Q_{e}(t)$ is the 2-norm of responses from the experiment. Note that $N$ controls tightness of the bounds and also the number of LS-DYNA solutions required.

The use of norms, although convenient, tends to hide the spatial relationships that exist between responses at different locations in the model. In order to study this spatial multi-dimensional dependency explicitly, a different metric must be established. Work by Anderson et al. (Ref.31] and Horta et al. (Ref.32 proposed the application of singular value decomposition derived basis vectors, or impact shapes. In this approach, time histories from analysis or experiments can be decomposed as;

$$
y(x, t)=\sum_{i=1}^{n} \sigma_{i} \phi_{i}(x) g_{i}(t)
$$

In this form, the impact shape vector $\phi_{i}$ sized $m \times 1$ contains the spatial distribution information for $m$ sensors, $g(\mathrm{t})$ contains the time modulation information, $\sigma$ contains scalar values with shape participation factors, and $n$ is the number of impact shapes to be included in the decomposition, often truncated based on allowable reconstruction error. Although Eq. (2) is written in continuous time form, for most applications, time is sampled at fixed intervals such that $t=k \Delta T$ where the integer $k=0, \ldots, L$ and $\Delta T$ is the sample time. In the discrete form of Eq. (2), singular value decomposition is used to recover the basis functions $(\varphi, \sigma$, and $g)$. The fractional contribution of the $f^{\text {th }}$ impact shape to the total response is proportional to $\delta_{i}$, defined as;

$$
\delta_{i}=\sigma_{i} / \sum_{l=1}^{n} \sigma_{l}
$$

Impact shapes can now be used to compare models using orthogonality. Orthogonality, computed as the dot product operation of vectors (or matrices), quantifies the projection of one vector onto another. If the projection is zero, vectors are orthogonal, i.e., distinct. This same idea applies when comparing test and analysis impact shapes. Numerically, the orthogonality metric is computed as;

$$
M_{2}=\breve{\Phi}^{T} \breve{\Psi}
$$

where $\breve{\Phi}$ is sized $m \times$ / with / measured impact shapes at $m$ locations and $\breve{\Psi}$ sized $m \times$ / are shapes computed using simulation data. Note that both $\breve{\Phi}$ and $\breve{\Psi}$ are normalized matrices such that $\breve{\Phi}^{T} \breve{\Phi}=/$ and $\breve{\Psi}^{T} \breve{\Psi}=/$. Because individual impact shape vectors are stacked column-wise, metric $M_{2}$ is a matrix sized / $\mathrm{x} /$ with diagonal values corresponding to the vector projection numerical value. If vectors are identical then their projection equals 1. Consequently, when evaluating models, multi-dimensional closeness with experiment is judged based on similarity of impact shapes and shape contributions.

If the model can be reconciled based on both time and spatial calibration metrics, a parameter set is computed which minimizes the squared sum of the prediction error, as defined in Eq. (5). The matrix $W$ is used to scale or remove data points from the time history.

$$
J=\sum_{k=1}^{L-1}\left(y(k \Delta T)-y_{e}(k \Delta T)\right)^{T} W\left(y(k \Delta T)-y_{e}(k \Delta T)\right)
$$

Optimization is based on the Constrained Optimization using Response Surface (CORS) scheme developed by Regis and Shoemaker (Ref. 33). Specifically, the algorithm starts by looking for parameter values away from the initial set of LS-DYNA solutions, then slowly steps closer to known solutions by solving a series of local constrained optimization problems. This optimization process will produce a global optimum if enough steps are taken. Of course, the user controls the number of steps and therefore the accuracy and computational expense in conducting the optimization. In cases where the predictive capability of the surrogate model is poor, CORS adds solutions in needed areas. Because parameter uncertainty is not used explicitly in the optimization, this approach is considered to be deterministic. If a probabilistic approach was used instead, in addition to a reconciling set, the user 
should also be able to determine the probability that the parameter set found is correct. These metrics will now be used to analyze the data from the second test without the DEA.

\section{Test/Analysis Results- Crash Test without DEA}

Initial runs of the system integrated FEM following the crash test without the DEA revealed key shortcomings in the FEM that were not evident when validating against the crash test with DEA. During the test, much of the subfloor secondary structure, including the keel beam and frames, exhibited structural failure. Pilot and co-pilot seat boxes were permanently deformed, and seat frames either buckled or failed in bending. The tail deformed significantly, and the forward swing cable fixture became dislodged at its interface to the bulkhead. Acceleration magnitudes increased by a factor of three, and pulse durations were reduced to around $40 \mathrm{~ms}$. The pulses were triangular in shape, but contained differing peaks depending on airframe location.

Results from the airframe FEM showed that the subfloor mesh was too coarse to accurately capture the keel beam and subfloor frame deformation. The predicted tail deformation was overly compliant compared with the observed tail deformation, and this behavior was attributed to a lack of detail, such as missing frames and stiffeners. Runtime failures occurred due to instabilities in the LSTC ATD FEMs. The subfloor and tail were modified in terms of detail and mesh refinement which resulted in an increased number of elements from 134,000 to 250,000.

Despite the improvements in the FEM and better agreement in deformation shapes, there were inconsistencies in the accelerometer data. These inconsistencies are highlighted when examining measured and predicted pilot seat box and floor vertical accelerations in Figure 12. The pilot seat box and floor acceleration pulse shapes and magnitudes both differ between test and analysis. Furthermore, test data show an abrupt spike of 60 to $70 \mathrm{~g}$ on the seat box and the floor, most likely from buckling and failure of the keel beam and shear panel under the seat box. This peak is not evident for the predicted seat box response which is approximately $30 \mathrm{~g}$. There is a spike of nearly $50 \mathrm{~g}$ in the predicted floor response, but the timing is not coincident. For simplicity, the shell thicknesses are considered constant over the whole region which represents a smeared effective stiffness, whereas the actual hardware has edge doublers and rivets and small cutouts. These simplifying assumptions in the FEM may not account for multiple thin shell buckling and failure modes. This lack of model fidelity is further illustrated by comparing the post-test pilot subfloor photographs to the analysis deformations in Figure 13. From the analysis, plastic deformation occurs along the shear panel and no failure is seen of the keel beam. The post-test photograph indicates a substantial rippled region of the keel beam forward of the shear panel.

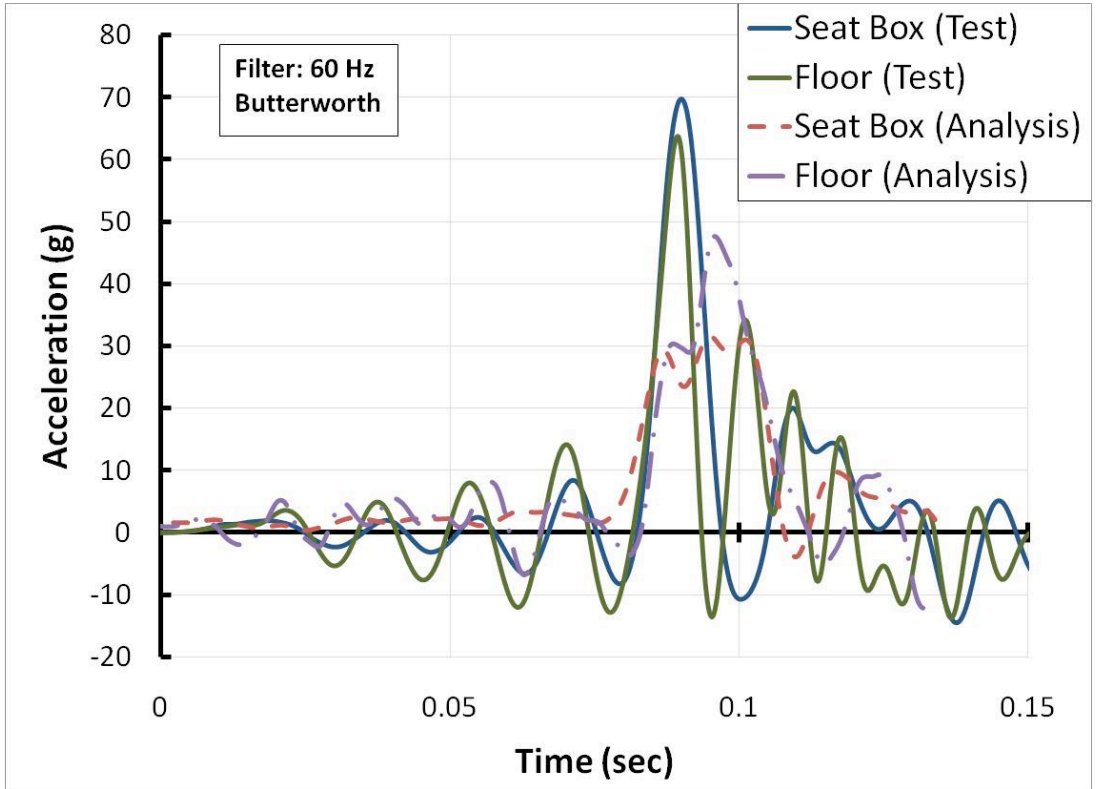

Figure 12. Comparison of test and analysis, pilot seat box and floor vertical acceleration, crash test without DEA 


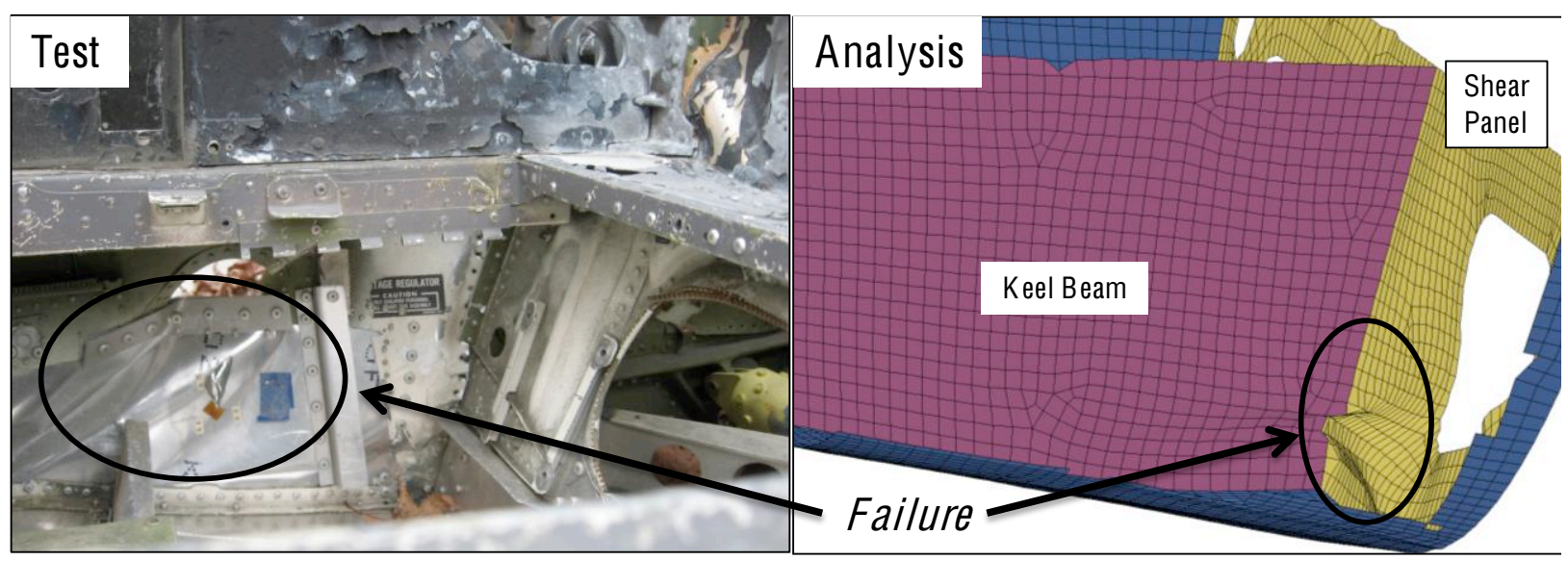

Figure 13. Pilot subfloor: post-test photograph versus analysis

The passenger floor accelerometer is mounted on a relatively stiff interface; thus, local effects are not introduced and the filtered test data tracks better. Comparisons of the passenger floor accelerations are shown in Figure 14. In this case, the passenger floor acceleration compares well in pulse shape and arrival time, but not in magnitude. Furthermore, the predicted pulse shape for the passenger floor is similar to the predicted pilot seat box and pilot floor pulse shapes.

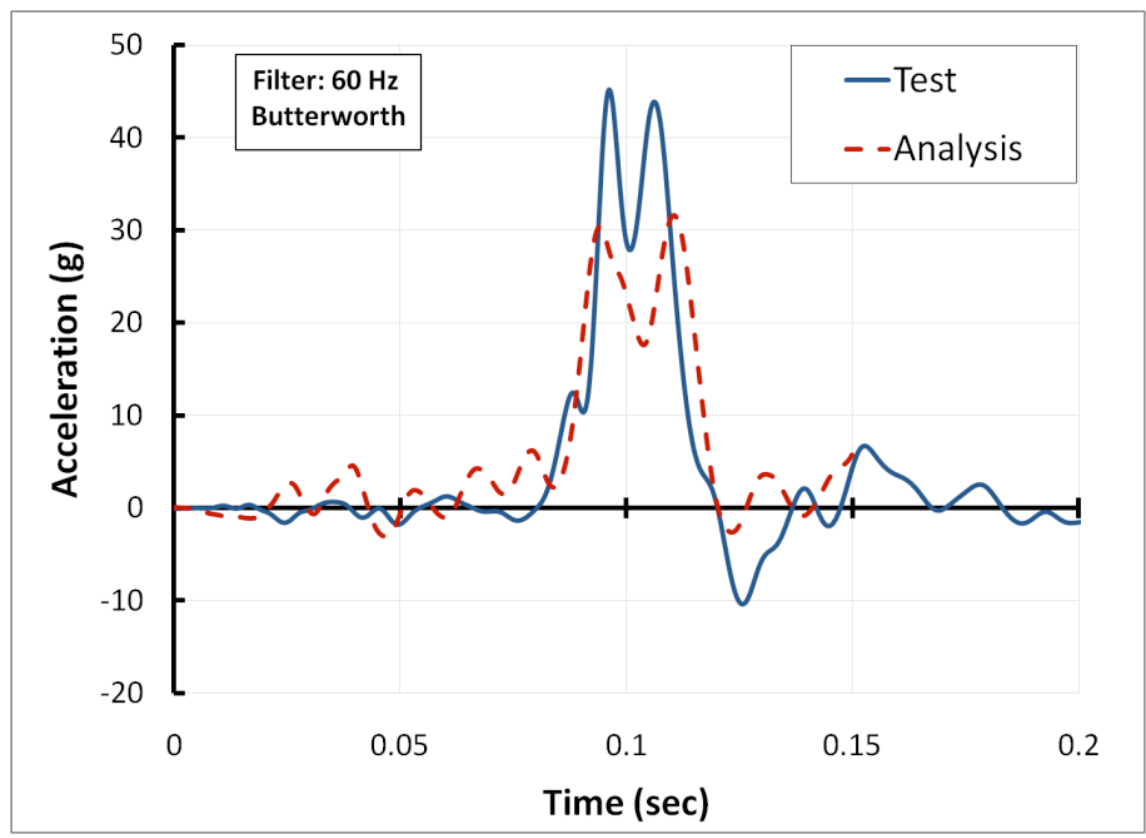

Figure 14. Comparison of test and analysis, passenger floor, vertical acceleration, crash test without DEA

Since the DEA significantly attenuated the impact response during the first crash test, this test data may not have been sufficient to validate the analytical model for the subsequent, more severe impact test. The airframe FEM acceleration responses were low and generally in the linear elastic range. The DEA acted as an isolator, accurately imparting loads into the airframe while obscuring deficiencies in the airframe model. These deficiencies became apparent when severe loads and highly nonlinear responses were introduced for the second full-scale test. There are multiple modes of failure in the test without the DEA which may or may not need to be represented by the FEM. From the standpoint of matching acceleration magnitudes and pulse shapes, the FEM detail is adequate in the 
passenger region, but questionable for the pilot region. The previous assertion of a "validated" FEM based on the crash test with the DEA was questioned. To determine whether more physical detail is required in the model or modification of existing parameters was sufficient to accurately capture responses, the comprehensive calibration described previously was performed.

The model calibration approach for the test without the DEA followed the approach described earlier. In this approach, a nominal parameter set was selected and LS-DYNA simulations were performed while varying the parameters. Selection of the parameters for the uncertainty study addresses three distinct aspects of the model: initial conditions (parameters 1 through 4), stiffness (parameters 5 and 6), and plasticity (parameter 7). Data from photogrammetric measurements is used to ascertain the attitude and velocity at impact. Slight changes in velocity and attitude result in significant changes in the magnitude of the kinetic energy. During uncertainty assessments, both vertical and horizontal velocities were modified directly, whereas attitude was changed by reorienting the normal vector which defines the rigid wall impact surface. These normal vector changes corresponded to ground pitch angles of $+/-1.5$ deg and +2 deg roll. To change the effective in-plane and bending stiffness of the secondary structure such as doublers or stiffeners, either the modulus of elasticity or the thickness defined in the shell property can be modified. For this study, the thickness was modified. Because the airframe is a combination of different alloys of thin sheet aluminum, the initial yield stress was used as a parameter in the subfloor where extensive damage occurs. All of the selected parameters and uncertainty ranges are listed in Table 2. A total of 97 simulations were conducted in LS-DYNA while varying parameters over their prescribed ranges using Halton sampling (Ref. 24.

Table 2. Parameter Description

\begin{tabular}{|c|c|c|c|c|c|}
\hline No. & $\begin{array}{l}\text { Parameter } \\
\text { Description }\end{array}$ & Nominal & $\begin{array}{l}\text { Lower } \\
\text { Bound }\end{array}$ & $\begin{array}{l}\text { Upper } \\
\text { Bound }\end{array}$ & $\begin{array}{c}\text { Calibrated } \\
\text { Values }\end{array}$ \\
\hline 1 & Horizontal Velocity (ft/s) & 38 & 37 & 40 & 37.2 \\
\hline 2 & Vertical Velocity (ft/s) & 26 & 23 & 27 & 27 \\
\hline 3 & Pitch Vector Change (in) & 0 & -0.052 & 0.052 & 0.036 \\
\hline 4 & Roll Vector Change (in) & 0 & 0 & 0.07 & 0.07 \\
\hline 5 & Belly thickness (in) & 0.09 & 0.08 & 0.1 & 0.08 \\
\hline 6 & Keel Beam Thickness (in) & 0.04 & 0.03 & 0.05 & 0.03 \\
\hline 7 & Subfloor initial yield stress (psi) & 45,000 & 30,000 & 60,000 & 30039 \\
\hline
\end{tabular}

If a solution exists within the parameter set that would reconcile test with analysis, then optimization can be applied. If a solution does not exist, the parameter set must be revisited or more detail is required in the FEM. To construct the uncertainty bounds, for each of the 97 runs, the velocity 2-norm metric $Q(t, p)$ was computed with velocities at 37 sensors. Velocity was selected to compute the metric because high frequency effects are naturally filtered. In Figure 15, the 2-norm analysis bounds - and _ are plotted as a function of time in dashed blue and the test $Q(t, p)$ is in solid-red. With this sample size, the probability of being able to reconcile test with analysis during times when test results are outside the analysis bounds is less than -. Except for time values near $0.085 \mathrm{sec}$, this parameter set provided enough freedom to adjust the model for calibration. 


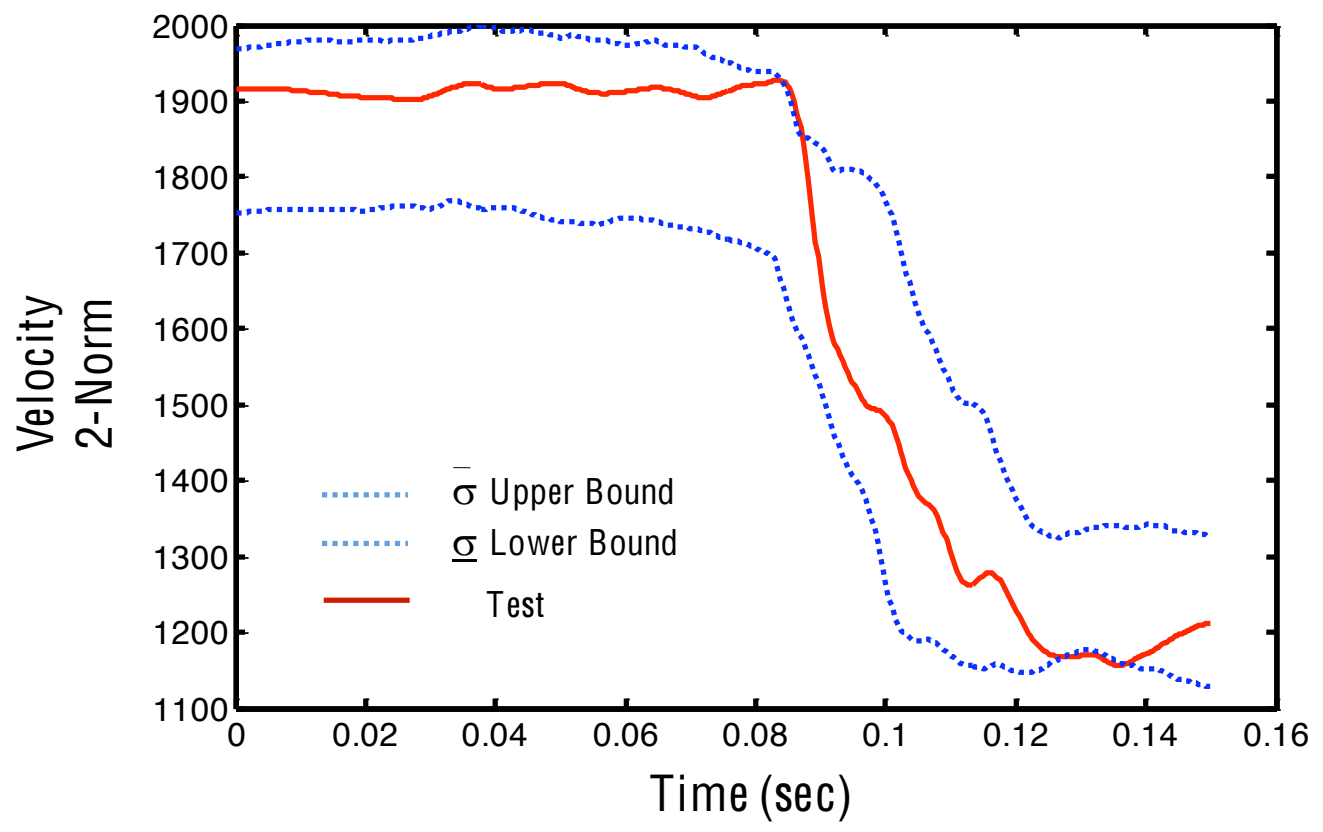

Figure 15. Uncertainty bounds for velocity 2-norm, analysis (dashed blue) versus test (solid red).

Individually altering parameters without any guidance as to the importance of those parameters can be time consuming and inefficient. Therefore, contribution of a single parameter variance to the total variance was computed (Refs. 25 and 27] to determine whether parameters should be retained or removed from the solution response set. Figure 16 shows the individual parameter contributions to the variance of the velocity 2-norm. Only first order variance effects are shown. Within the critical time frame of belly impact to rebound (0.08 to 0.15 seconds), the parameters with the highest contributions to variance are the horizontal velocity, vertical velocity and pitch normal. Other parameters such as belly thickness, keel thickness, and yield stress contribute significantly less.

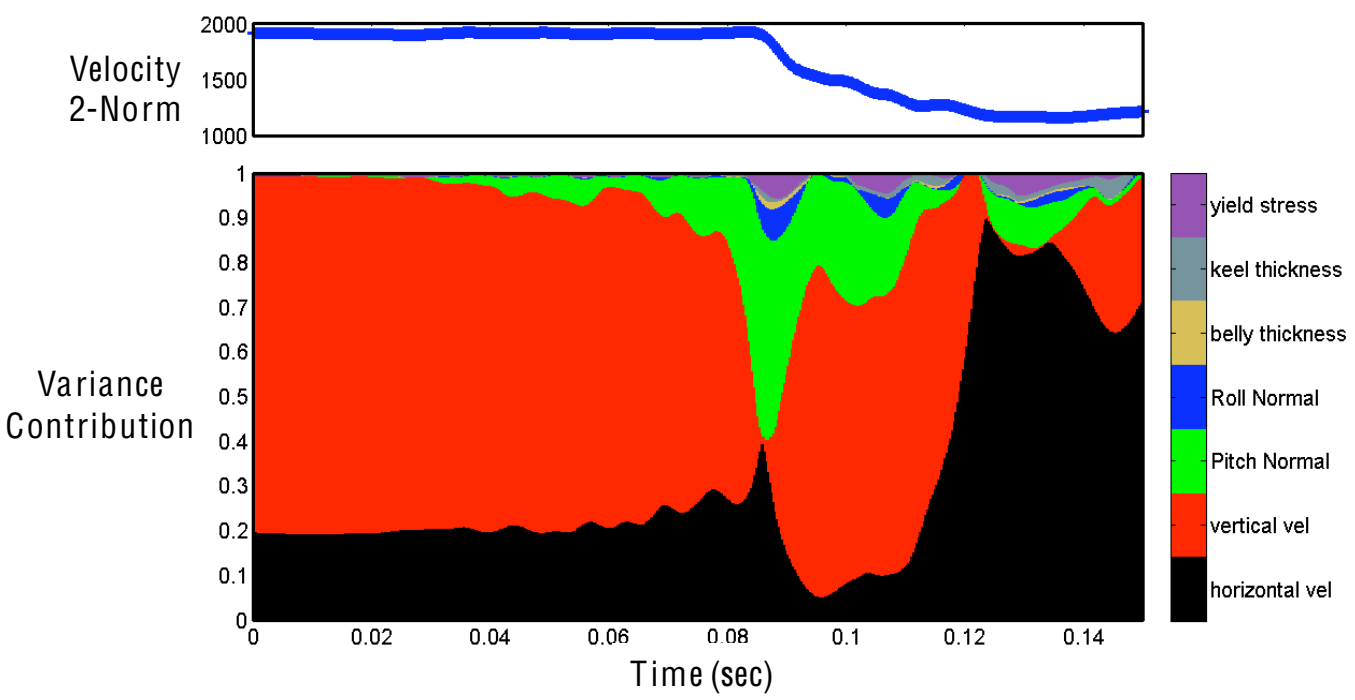

Figure 16. Parameter sensitivity. 
Graphical point-by-point time-history comparisons for velocity, while at first practical, are not entirely definitive for quantifying error if the model response is very complex. Instead, orthogonality of impact shapes is also used as a multi-dimensional calibration metric. To compute impact shapes, time responses were decomposed into dominant deformation shapes using $\delta_{i}$ as defined in Eq. (3). Test data was processed similarly and orthogonality of test and analysis impact shapes was then computed. Figure 17 shows the orthogonality results using velocity output. The first five shapes and the percentage value of each shape's contribution are plotted for LS-DYNA along the ordinate and test along the abscissa. Two dominant basis vectors contribute almost $89 \%$ of the LS-DYNA response and $85 \%$ of the test response. The orthogonality values are 0.99 for the first basis vector and 0.68 for the second. These two basis vectors are related to the two rigid body modes in the vertical and horizontal directions. The remaining three basis vectors can be considered airframe deformable modes and differ between test and analysis.

Finally, calibration of the model using optimization was attempted, starting with the original 97 LS-DYNA runs, to minimize the squared sum prediction error difference (see Eq. (5)) between the model and test velocity. The weighting function was selected to emphasize the post-impact behavior. Because of time constraints, only 20 additional LS-DYNA runs were executed during the optimization. Parameter values for the calibrated set are shown in the last column of Table 2. Figure 18 shows a comparison of the velocity 2-norm for the test, baseline, and calibrated model. Prediction error values of the 2-norm for velocities are reduced by $51 \%$; however, from the parameter table, note that four out of the seven parameters are near their bounds. This result is often an indication of problems with the parameter selection. Improvements from this calibration effort are minimal in terms of velocity 2-norm. Orthogonality results with the calibrated model, not shown here, show a slight improvement in the impact shape contribution for the first two modes with no appreciable change in the higher impact shapes.

Two issues were identified following this first attempt at calibration. First, the parameters with the high contribution to the total variance are primarily test initial conditions which only affected the first two impact shapes. Second, the structural parameters identified for calibration have a relatively small influence on the total variance. Hence, to correct this problem, the parameter set must be re-visited. Subsequent calibration of model parameters without initial conditions in the parameter set should improve the effectiveness of the system-integrated finite element model.

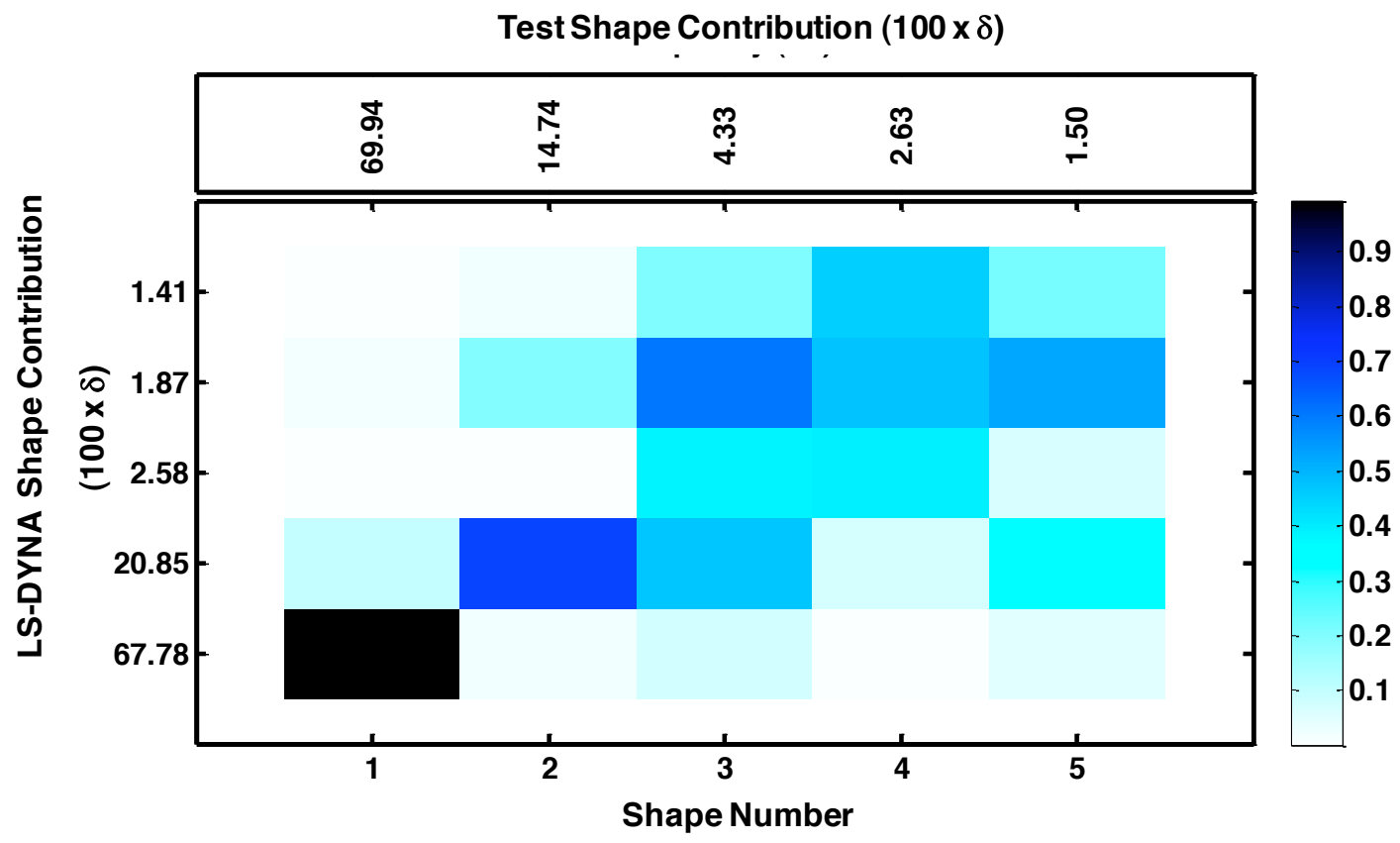

Figure 17. Orthogonality of test versus analysis basis vectors 


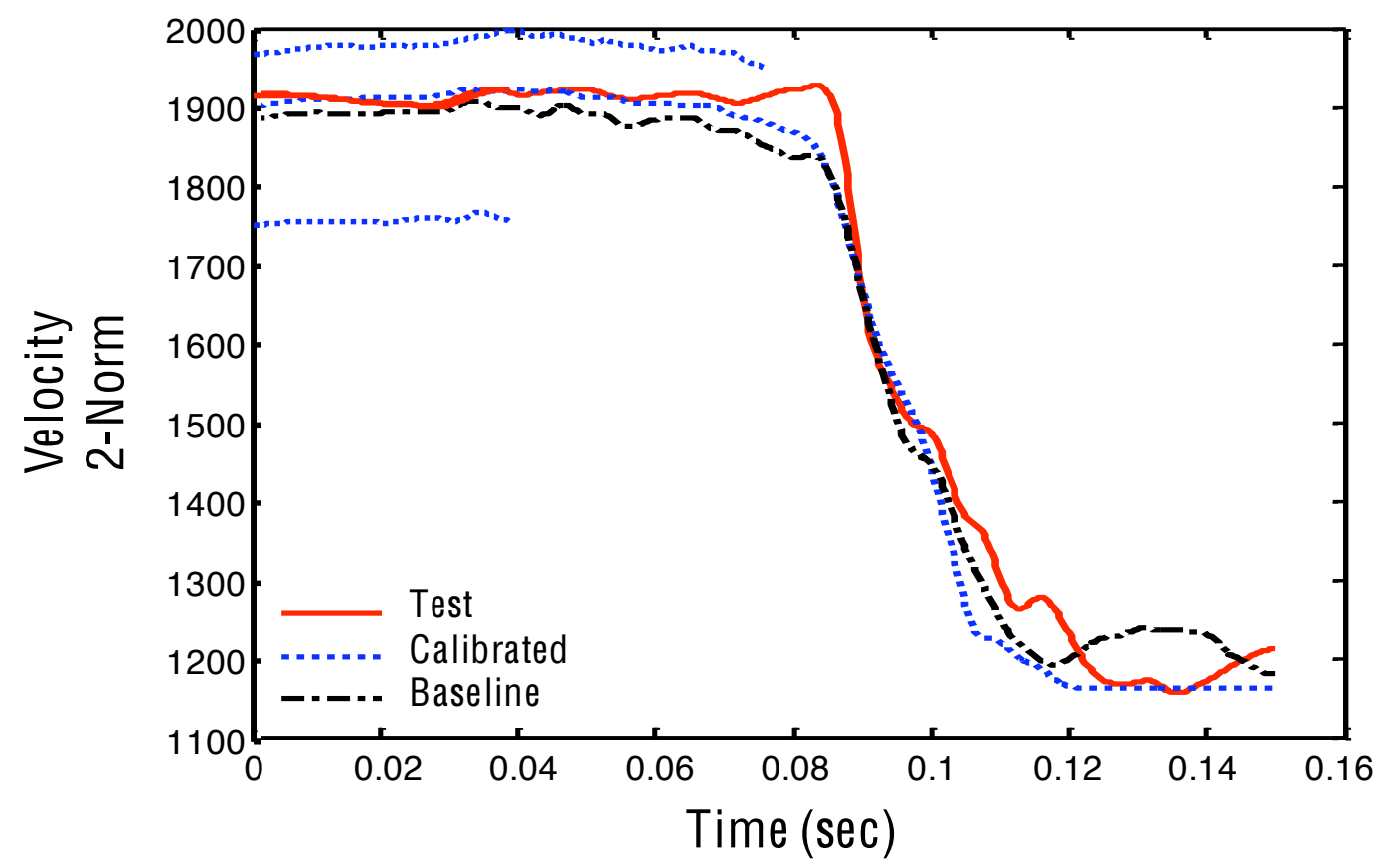

Figure 18. Comparison of velocity 2-norm for baseline, test, and calibrated model

\section{Concluding Remarks}

Results from two full-scale helicopter crash tests have been presented. In the first test a helicopter was fitted with a Deployable Energy Absorbing (DEA) system, and the second test was conducted without the DEA. Both tests were sponsored by the NASA Subsonic Rotary Wing Program in an effort to evaluate new materials and structural concepts to improve rotorcraft crashworthiness and to increase occupant survivability. The tests demonstrated a peak acceleration reduction upon impact by a factor of three when using the DEA.

Models to predict the overall performance of the system were developed and compared to test. Acceleration time histories at the pilot seat box location and the floor were compared to analysis for both crash tests. Reasonable agreement was seen between test and analysis for the crash test performed with the DEA. The accelerations waveforms and peak values were significantly different between test and analysis for the crash test performed without the DEA. One reason for this discrepancy is the fact that acceleration levels for the test with the DEA were significantly lower and therefore less energy went into deforming the fuselage. Consequently, model fidelity played less of a factor for the test with the DEA.

LS-DYNA model calibration was performed based on two new calibration metrics: (1) a 2-norm velocity bound metric, and (2) orthogonality of test and analysis impact shapes. Results with metric (1) were used to assess the probability of reconciling test with analysis after uncertainty propagation studies. An initial attempt to calibrate the full-scale model resulted in a calibrated set of parameters that reduced the overall 2-norm velocity prediction error by $50 \%$ but did not improve orthogonality of test with analysis. Sensitivity results indicated that impact conditions such as vertical and horizontal velocities had the highest contribution to the total response variance. This finding tended to overshadow changes in structural parameter values needed to improve the orthogonality results.

\section{References}

${ }^{1}$ Jackson, K.E., Fuchs, Y. T., and Kellas, S., "Overview of the NASA Subsonic Rotary Wing Aeronautics Research Program in Rotorcraft Crashworthiness," Journal of Aerospace Engineering, Special Issue on Ballistic Impact and Crashworthiness of Aerospace Structures, Volume 22, No. 3, July 2009, pp. 229-239.

${ }^{2}$ Kellas, S., "Deployable Rigid System for Crash Energy Management," U.S. Patent Nos. 6,755,453 on June 29, 2004; 6,976,729 on December 20, 2005; and 7,040,658 on May 9, 2006. 
${ }^{3}$ Wittlin, G. and Gamon, M.A.: "Experimentally Verified Analytical Techniques for Predicting Vehicle Crash Responses," Proceedings of the AIAA $11^{\text {th }}$ Annual Meeting and Technical Display, AIA A Paper No. 75-273, Feb. 1975.

${ }^{4}$ Cronkhite, J.D., and Mazza, L.T.: "KRASH Analysis Correlation with the Bell ACAP Full-Scale Aircraft Crash Test," Proceedings of the National Technical Specialists' Meeting on Advanced Rotorcraft Structures: Requirements Vs. Opportunities, a Look at the Future. V A, Oct. 1988.

${ }^{5}$ Jackson, K. E., Fasanella, E. L., and Boitnott, R. L., "Full-Scale Crash Test and Finite Element Simulation of a Composite Prototype Helicopter," N ASA/TP-2003-212641, 2003.

${ }^{6}$ Wittlin, G., Smith, M., and Richards, M., "Airframe Water Impact Analysis Using a Combine MSC/DYTRANDRI/KRASH Approach," Proceedings of the American Helicopter Society 53rd Annual Forum, Virginia Beach, C A, 29 April- 1 May 1997.

${ }^{7}$ Hallquist, John Q., “LS-DYNA Keyword User's Manual,” Version 971, Revision 5.0, Livermore Software Technology Company, Livermore, CA, May 2010.

${ }^{8}$ Jackson, K. E., and Fasanella, E. L., "Crash Simulation of a Vertical Drop Test of a Commuter-Class Aircraft," International Journal of Crashworthiness, 1754-2111, Vol. 10, Issue 2, 2005, pp. 173 - 182.

${ }^{9}$ Annett, M. S., Polanco, M. A., "System-Integrated Finite Element Analysis of a Full-Scale Helicopter Crash Test with Deployable Energy Absorbers," Proceedings of the American Helicopter Society $66^{\text {th }}$ Annual Forum, Phoenix, AZ, 11-13 May, 2010.

${ }^{10}$ Anonymous, Military Standard, MIL-STD-1290A (AV), Light Fixed- and Rotary-Wing Aircraft Crash Resistance, Department of Defense, Washington DC, 20301, 26 September 1988.

${ }^{11}$ Anonymous, Preliminary Rotary Wing Full Spectrum Crashworthiness Criteria, v5.0. 3 January 2011.

${ }^{12}$ AIAA. Guide for the Verification and Validation of Computational Fluid Dynamics Simulations, American Institute of Aeronautics and Astronautics, AIA A-G-077-1998, Reston, V A, 1998.

${ }^{13}$ Roberts, J. C., Merkle, A., Biermann, P., Ward, E., Carkhuff, B., and Cain, R. P. "Computational and Experimental Models of the Human Torso for Non-Penetrating Ballistic Impact," Journal of Biomechanics, Vol. 40, 2007 pp. $125-136$.

${ }^{14}$ Littell, J. D., "A Comparative Analysis of Two Full Scale MD-500 Helicopter Crash Tests,", Proceedings of the 2010 SEM Annual Conference \& Exposition on Experimental and Applied Mechanics, Uncasville, CT, June 2011 (submitted for publication).

${ }^{15}$ Anonymous, Structural Repair Manual, CSP-SRM-6, MD Helicopters, Inc, Mesa, AZ, May 2006.

${ }^{16}$ Polanco, M., "A Parametric Study on a Shell-Based Model of a Kevlar/Epoxy Composite Honeycomb," AHS Technical Specialists Meeting on Rotorcraft Structures and Survivability, Williamsburg, V A, 27-29 0ctober, 2009.

${ }^{17}$ Guha, S., Bhalsod, D., and Krebs, J., "LSTC Hybrid III Dummies, Positioning \& Post-Processing, Dummy Version: LSTC.H3.103008_v1.0," LSTC Michigan, October 30, 2008

${ }^{18}$ Tabiei, A., Lawrence, C. and Fasanella, E., "Validation of Finite Element Crash Test Dummy Models for Predicting Orion Crew Member Injuries During a Simulated Vehicle Landing," NASA/TM-2009-215476, 2009.

${ }^{19}$ Polanco, M. A., and Littell, J., "Vertical Drop Testing and Simulation of Anthropomorphic Test Devices," Proceedings of the American Helicopter Society 67th Annual Forum, Virginia Beach, V A, May 2011 (submitted for publication).

${ }^{20}$ Eiband, M. A., "Human Tolerance to Rapidly Applied Accelerations: A Summary of the Literature," N ASA Memorandum 5-19-59E, June 1959

${ }^{21}$ Brinkley, J.W and Shaffer, J. T., "Dynamic Simulation Tecniques for the Design of Escape Systems: Current Applications and Future Air Force Requirements," Symposium on Biodynamic Models and their Applications, AMRL-TR-71-29, WrightPatterson Air Force Base. Dayton, OH : Aerospace Medical Research Laboratory, 1970.

${ }^{22}$ Brinkley, J. W., Specker, L. J.,and Mosher, S. E., "Development of Acceleration Exposure Limits for Advanced Escape Systems," NATO AGARD Proceedings, AGARD-CP-472, 1990.

${ }^{23}$ Horta, L. G., Reaves, M. C., Annett, M. S., Jackson, K. E., "Multi-Dimensional Calibration of Impact Dynamic Models," Proceedings of the IMAC-XXIX Conference and Exposition on Structural Dynamics, Jacksonville, FL, 31 January-3 February, 2011.

${ }^{24}$ Halton, J.H., "On the Efficiency of Certain Quasi-Random Sequences of Points in Evaluating Multi-Dimensional Integrals." Numerische Mathematik, Vol. 2, pp. 84-90, 1960.

${ }^{25}$ Sobol, I.M., Tarantola, S., Gatelli, D., Kucherenko, S.S, and Mauntz, W.: "Estimating Approximation Error When Fixing Unessential Factors in Global Sensitivity Analysis." Reliability Engineering and System Safety 92, 2007, pp. 957-960.

${ }^{26}$ Mullershon, H., and Liebsher, M.: "Statistics and Non-Linear Sensitivity Analysis with LS-OPT and DSPEX." Proceedings of the $10^{\text {th }}$ International LS-DYNA Users Conference, Dearborn, MI, 8-10 June, 2008, pp. 4-1,4-13.

${ }^{27}$ Homma, T, and Saltelli, A., "Importance Measures in Global Sensitivity Analysis of Nonlinear Models." Reliability Engineering and System Safety 52, 1996, pp. 1-17.

${ }^{28}$ Sudret, B., "Global Sensitivity Analysis Using Polynomial Chaos Expansion." Reliability Engineering and System Safety 93 (2008), pp. 964-979.

${ }^{29}$ Mullur, A. and Messac, A., "Extended Radial Basis Functions: More Flexible and Effective Metamodeling." $A / A A$ Journal, Vol., 43, No. 6, June 2005.

${ }^{30}$ Mullur, A. and Messac, A.: "Metamodeling Using Extended Radial Basis Functions: A Comparative Approach." Engineering with Computers (2006) ,Vol. 21: pp.203-217. 
${ }^{31}$ Anderson, M.C., Gan, W., and Hasselman, T.K., "Statistical Analysis of Modeling Uncertainty and Predictive Accuracy for Nonlinear Finite Element Models." Proceedings of the $69^{\text {th }}$ Shock and Vibration Symposium, Minneapolis/St. Paul, MN, 1998.

${ }^{32}$ Horta, L.G., Lyle, K.H., Lessard, W.B., "Evaluation of Singular Value Decomposition Approach for Impact Dynamic Data Correlation." NASA TM 2003-212657, 0ct. 2003.

${ }^{33}$ Regis, R.G., and Shoemaker, C.A., "Constrained Global Optimization of Expensive Black Box Functions Using Radial Basis Functions.” Journal of Global Optimization (2005), Vol. 31: pp. 153-171, Sept. 2005. 